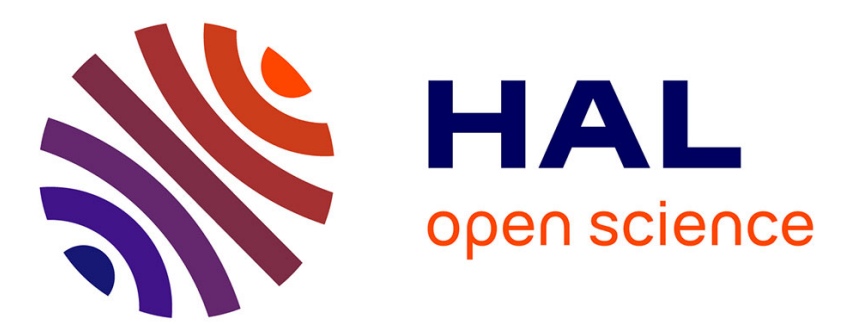

\title{
Finite element implementation of nearly-incompressible rheological models based on multiplicative decompositions
}

\author{
Stéphane Lejeunes, Adnane Boukamel, Stéphane Meo
}

\section{To cite this version:}

Stéphane Lejeunes, Adnane Boukamel, Stéphane Meo. Finite element implementation of nearlyincompressible rheological models based on multiplicative decompositions. Computers \& Structures, 2011, 89 (3-4), pp.411-421. 10.1016/j.compstruc.2010.11.013 . hal-00551691

\author{
HAL Id: hal-00551691 \\ https://hal.science/hal-00551691
}

Submitted on 1 Jan 2018

HAL is a multi-disciplinary open access archive for the deposit and dissemination of scientific research documents, whether they are published or not. The documents may come from teaching and research institutions in France or abroad, or from public or private research centers.
L'archive ouverte pluridisciplinaire HAL, est destinée au dépôt et à la diffusion de documents scientifiques de niveau recherche, publiés ou non, émanant des établissements d'enseignement et de recherche français ou étrangers, des laboratoires publics ou privés. 


\title{
Finite element implementation of nearly-incompressible rheological models based on multiplicative decompositions
}

\author{
S. Lejeunes ${ }^{*, a}$, A. Boukamel ${ }^{\mathrm{a}, \mathrm{b}}$, S. Méo ${ }^{\mathrm{c}}$ \\ ${ }^{a}$ Laboratoire de mécanique et d'acoustique de Marseille (CNRS UPR7051), 31 chemin Joseph-Aiguier, 13402 Marseille cedex 20, France \\ ${ }^{b}$ Ecole Centrale Marseille, IMT/Technopôle Chateau-Gombert, 13451 Marseille Cedex 20, France \\ ${ }^{c}$ Laboratoire de Mécanique et Rhéologie (Université de Tours), BP 407, F37204 Cedex 3, Tours, France
}

\begin{abstract}
The present work is concerned with the numerical integration of finite viscoelastic or viscoplastic models. A numerical integration scheme based on the definition of a flow direction and a flow amplitude as in elastoplasticity is proposed. The most original feature of this approach resides in a local correction of the direction and amplitude with a sub-stepping strategy. Comparisons with the results obtained using a classical tensorial integrator based on a Runge-Kutta-Fehlberg scheme are provided. The reliability of the present numerical scheme is investigated with three rheological models two are viscoelastic (Zener and Poynting-Thomson) and one is viscoplastic.
\end{abstract}

Key words: finite strain, viscoelasticity, viscoplasticity, finite elements, integration scheme.

\section{Introduction}

Many rheological models based on the fundamental principles of thermodynamics and the multiplicative decomposition of the strain gradient have been developed during the last twenty years, for simulating the behavior of dissipative materials at large strains. These models are often an extension of small strain ones and in many cases a numerical implementation has been proposed. However, these numerical schemes are not always discussed in terms of their reliability (robustness, convergence, stability) and even less in terms of their numerical performances. As the complexity of these models continued to increase, there is still a need for robust, efficient and generic numerical schemes.

This paper focuses on a class of rheological models based on internal variables and evolution equations. An interesting idea in this framework consists in decomposing the internal variable rates in terms of direction and magnitude. This is a classical procedure in elasto-plasticity where an objective rate tensor of plastic deformation is decomposed in this way. In the case of associative plasticity with J2 flow, the problem reduces to the determination of a scalar parameter. This process is known as the radial-return algorithm (see for instance $[1,2]$ for a finite strain implementation and the references therein). In the case of viscoelasticity, the evolution of the internal variables is generally fully tensorial and cannot therefore be reduced to a scalar equation. Many authors have developed tensorial integrators which are based on a generalization of standard integration schemes. For example, [3] have used a Backward-Euler scheme in the framework of the Poynthing-Thomson model, and [4] used an explicit Runge-Kutta scheme with the same model. The main difficulty encountered with this schemes arises from the determination of the consistent tangential operator, which is not a straightforward task. In the framework of a generalized Maxwell model, [5] proposed an application of diagonal implicit Runge Kutta methods (DIRK). In this case, the discrete equilibrium equations and evolution laws are assembled in a global differential algebraic system, which is solved by an implicit Runge Kutta algorithm. This

\footnotetext{
${ }^{*}$ Corresponding author

Email addresses: lejeunes@lma.cnrs-mrs.fr, tel: 033491054382 ,fax: 033491054749 (S. Lejeunes), boukamel@centrale-marseille.fr(A. Boukamel), stephane.meo@univ-tours.fr(S. Méo)
} 
method is robust and generic but it involves solving the evolution laws at the global level.

Besides the class of tensorial integrators, some authors have proposed specific algorithms based on exponential mapping methods (see [6]). In the paper of [7], this technique was developed on a Zener model: by setting the evolution law in the principal space and using logarithmic principal stretches, the integration was reduced to determining a non-linear system of three equations, using a local newton scheme. This scheme is very robust in the case of viscoelastic and viscoplastic models, and since the earlier paper by [7], many authors have used this strategy (see for example $[8,9,10,11])$. For elastoplasticity, [12] have proposed an exponential mapping with a full tensorial integration and a consistent linearization. However, these algorithms are approximate ones since the evolution equations cannot generally be expressed as a linear differential system, and it is therefore not easy to obtain an exponential solution for the time integration. In the case of Maxwell elements, this approximation seems to be valid, but it would be interesting to apply this algorithm to a more complex case such as the Poynting-Thomson model. In the case of a viscoplastic model, [13] have compared the use of a Backward-Euler scheme with that of an Exponential mapping, and the rate of convergence obtained with both schemes was identical.

The integration scheme presented here is based on the concepts used in return mapping algorithms. A flow direction (a normalized tensor) is defined and introduced in the evolution equations in order to obtain flow magnitude evolution equations. A vectorial non-linear differential system is obtained and integrated with a Backward Euler scheme. By linearizing this system an Exponential integrator is obtained. If necessary, the integration can be corrected using a sub-stepping strategy. The results obtained with the present integrator are compared with a full tensorial Runge-KuttaFehlberg (RKF45) scheme (see [14, 15]). Three different rheological behaviors are also tested in order to determine the efficiency of these schemes with evolution laws that exhibit different nature of non-linearity.

This paper is organized as follows: thermodynamics of finite strain with intermediate states is recalled and applied to three different models in the first section. The evolution laws of Maxwell and Poynting-Thomson models are derived and a finite viscoplastic model is described. In the second section, the variational formulation adopted is briefly described. The numerical integration schemes are presented in the third section. Lastly, the reliability and the numerical performances for each rheological models are discussed, giving some simple examples.

\section{Constitutive Equations}

\subsection{Some definitions}

In the context of thermodynamics of irreversible processes, the constitutive equations must fulfill the Clausius Duhem inequality, which takes the following form in the Eulerian configuration and in the isothermal case:

$$
\mathcal{D}_{\text {int }}=\boldsymbol{\sigma}: \mathbf{D}-J^{-1} \rho_{0} \dot{\psi} \geq 0
$$

where $\boldsymbol{\sigma}$ is the Cauchy stress, $\mathbf{D}$ is the Eulerian rate of deformation, $J=\operatorname{det} \mathbf{F}$ is the volume variation (and $\mathbf{F}$ is the deformation gradient), $\rho_{0}$ is the volumetric mass in the initial configuration, $\mathcal{D}_{\text {int }}$ is the internal dissipation and $\psi$ is the specific free energy. This paper focuses on rheological models based on the concept of intermediate states initially proposed by $[16,17]$. In line with [18], the deformation gradient is first split into volumetric and isochoric parts. The latter part is split into elastic and inelastic parts as shown in figure 1:

$$
\mathbf{F}=\left(J^{\frac{1}{3}} \mathbf{1}\right) \cdot \overline{\mathbf{F}}=\left(J^{\frac{1}{3}} \mathbf{1}\right) \cdot \overline{\mathbf{F}}_{\mathbf{e}} \cdot \overline{\mathbf{F}}_{\mathbf{i}}
$$

where $\overline{\mathbf{F}}$ is the incompressible part of the deformation gradient, $\overline{\mathbf{F}}_{\mathbf{e}}$ can be said to be an incompressible elastic deformation gradient and $\overline{\mathbf{F}}_{\mathbf{i}}$ is an incompressible inelastic deformation gradient. These choices imply that the non-elastic processes involved will be purely isochoric and all the volume changing deformation is restricted to be reversible. In what follows, it is assumed that the free energy is additively split into a volumetric, an equilibrium and a nonequilibrium part:

$$
\psi=\psi_{e q}(\mathbf{X})+\psi_{n e q}(\mathbf{Y})+\psi_{\text {vol }}(J)
$$




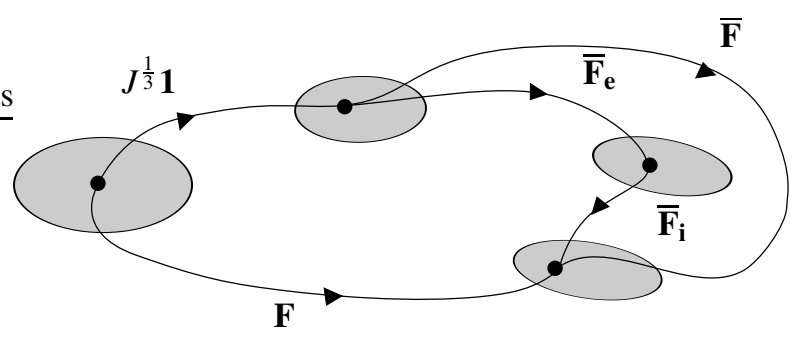

Figure 1: Split of the deformation gradient

where $\mathbf{X}$ and $\mathbf{Y}$ are one of the left Cauchy Green tensors: $\overline{\mathbf{B}}=\overline{\mathbf{F}} \cdot \overline{\mathbf{F}}^{\mathrm{T}}, \overline{\mathbf{B}}_{\mathbf{e}}=\overline{\mathbf{F}}_{\mathbf{e}} \cdot \overline{\mathbf{F}}_{\mathbf{e}}{ }^{\mathrm{T}}$ or $\overline{\mathbf{B}}_{\mathbf{i}}=\overline{\mathbf{F}}_{\mathbf{i}} \cdot \overline{\mathbf{F}}_{\mathbf{i}}^{\mathrm{T}}$, depending on the rheological model adopted. The time derivative of the free energy is therefore:

$$
\dot{\psi}=\frac{\partial \psi_{e q}}{\partial \mathbf{X}}: \dot{\mathbf{X}}+\frac{\partial \psi_{n e q}}{\partial \mathbf{Y}}: \dot{\mathbf{Y}}+\frac{\partial \psi_{v o l}}{\partial J} \dot{J}
$$

The time derivative of $J$ is

$$
\dot{J}=J(\mathbf{1}: \mathbf{L}) \quad \text { with } \quad \mathbf{L}=\dot{\mathbf{F}} \mathbf{F}^{-1}
$$

and the time derivative of the strain variables $\mathbf{X}$ and $\mathbf{Y}$ is given by two of the following:

$$
\begin{aligned}
& \dot{\overline{\mathbf{B}}}=\mathbf{L} \cdot \overline{\mathbf{B}}+\overline{\mathbf{B}} \cdot \mathbf{L}^{\mathrm{T}}-\frac{2}{3}(\mathbf{1}: \mathbf{D}) \overline{\mathbf{B}} \\
& \dot{\overline{\mathbf{B}}}_{\mathbf{i}}=\overline{\mathbf{L}}_{\mathbf{i}} \cdot \overline{\mathbf{B}}_{\mathbf{i}}+\overline{\mathbf{B}}_{\mathbf{i}} \cdot \overline{\mathbf{L}}_{\mathbf{i}}^{\mathrm{T}} \\
& \dot{\overline{\mathbf{B}}}_{\mathbf{e}}=\mathbf{L} \cdot \overline{\mathbf{B}}_{\mathbf{e}}+\overline{\mathbf{B}}_{\mathbf{e}} \cdot \mathbf{L}^{\mathrm{T}}-2 \overline{\mathbf{V}}_{\mathbf{e}} \cdot \overline{\mathbf{D}}_{\mathbf{i}}^{\mathbf{o}} \cdot \overline{\mathbf{V}}_{\mathbf{e}}-\frac{2}{3}(\mathbf{1}: \mathbf{D}) \overline{\mathbf{B}}_{\mathbf{e}}
\end{aligned}
$$

Here we introduce the objective Eulerian inelastic rate of deformation $\overline{\mathbf{D}}_{\mathbf{i}}^{\mathbf{0}}$ defined by

$$
\overline{\mathbf{D}}_{\mathbf{i}}^{\mathbf{0}}=\overline{\mathbf{R}}_{\mathbf{e}} \cdot \overline{\mathbf{D}}_{\mathbf{i}} \cdot \overline{\mathbf{R}}_{\mathbf{e}}^{\mathrm{T}}
$$

with $\overline{\mathbf{R}}_{\mathbf{e}}$ originates from the polar decomposition $\overline{\mathbf{F}}_{\mathbf{e}}=\overline{\mathbf{V}}_{\mathbf{e}} \cdot \overline{\mathbf{R}}_{\mathbf{e}}$ and $\overline{\mathbf{D}}_{\mathbf{i}}=\left(\dot{\overline{\mathbf{F}}} \cdot \overline{\mathbf{F}}_{\mathbf{i}}^{-1}\right)_{\text {sym }}$.

\subsection{Zener model}

This model is schematized in figure 2, and we have $\mathbf{X}=\overline{\mathbf{B}}, \mathbf{Y}=\overline{\mathbf{B}}_{\mathbf{e}}$. Based on (5), (6) and (8) the time derivative of the free energy is:

$$
\begin{aligned}
\dot{\psi}= & \frac{\partial \psi_{e q}}{\partial \overline{\mathbf{B}}}:\left(\mathbf{L} \cdot \overline{\mathbf{B}}+\overline{\mathbf{B}} \cdot \mathbf{L}^{\mathrm{T}}-\frac{2}{3}(\mathbf{1}: \mathbf{D}) \overline{\mathbf{B}}\right)+\frac{\partial \psi_{\text {neq }}}{\partial \overline{\mathbf{B}}_{\mathbf{e}}}:\left(\mathbf{L} \cdot \overline{\mathbf{B}}_{\mathbf{e}}+\overline{\mathbf{B}}_{\mathbf{e}} \cdot \mathbf{L}^{\mathrm{T}}-2 \overline{\mathbf{V}}_{\mathbf{e}} \cdot \overline{\mathbf{D}}_{\mathbf{i}}^{\mathbf{o}} \cdot \overline{\mathbf{V}}_{\mathbf{e}}-\frac{2}{3}(\mathbf{1}: \mathbf{D}) \overline{\mathbf{B}}_{\mathbf{e}}\right) \\
& +J \frac{\partial \psi_{v o l}}{\partial J}(\mathbf{1}: \mathbf{L})
\end{aligned}
$$

After some calculations, the following expression for the internal dissipation can be obtained:

$$
\begin{aligned}
\mathcal{D}_{\text {int }}= & \left(\boldsymbol{\sigma}-\rho_{0} J^{-1}\left(2 \overline{\mathbf{B}} \cdot \frac{\partial \psi_{e q}}{\partial \overline{\mathbf{B}}}\right)^{\mathrm{D}}-\rho_{0} J^{-1}\left(2 \overline{\mathbf{B}}_{\mathbf{e}} \cdot \frac{\partial \psi_{\text {neq }}}{\partial \overline{\mathbf{B}}_{\mathbf{e}}}\right)^{\mathrm{D}}-\rho_{0}\left(\frac{\partial \psi_{\text {vol }}}{\partial J} \mathbf{1}\right)\right): \mathbf{D} \\
& +\left(2 \rho_{0} J^{-1} \overline{\mathbf{V}}_{\mathbf{e}} \cdot \frac{\partial \psi_{\text {neq }}}{\partial \overline{\mathbf{B}}_{\mathbf{e}}} \cdot \overline{\mathbf{V}}_{\mathbf{e}}\right): \overline{\mathbf{D}}_{\mathbf{i}}^{\mathbf{o}} \geq 0
\end{aligned}
$$

where the superscript ${ }^{\mathrm{D}}$ stands for the deviatoric operator. For arbitrary choices of $\mathbf{D}$, the constitutive equations are derived as well as a remainder inequality governing the non-negativeness of the internal dissipation. The stress is defined as the sum of a deviatoric equilibrium, a deviatoric non-equilibrium and a spherical part:

$$
\boldsymbol{\sigma}=\overbrace{\left(2 \rho_{0} J^{-1} \overline{\mathbf{B}} \cdot \frac{\partial \psi_{e q}}{\partial \overline{\mathbf{B}}}\right)^{\mathrm{D}}}^{\boldsymbol{\sigma}_{e q}}+\overbrace{\left(2 \rho_{0} J^{-1} \overline{\mathbf{B}}_{\mathbf{e}} \cdot \frac{\partial \psi_{\text {neq }}}{\partial \overline{\mathbf{B}}_{\mathbf{e}}}\right)^{\mathrm{D}}}^{\boldsymbol{\sigma}_{\text {neq }}}+\overbrace{\rho_{0} \frac{\partial \psi_{\text {vol }}}{\partial J} \mathbf{1}}^{\boldsymbol{\sigma}_{\text {vol }}}
$$




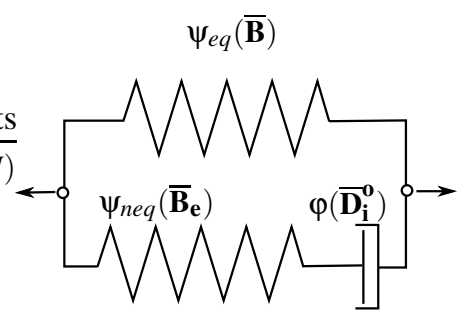

Figure 2: Zener model

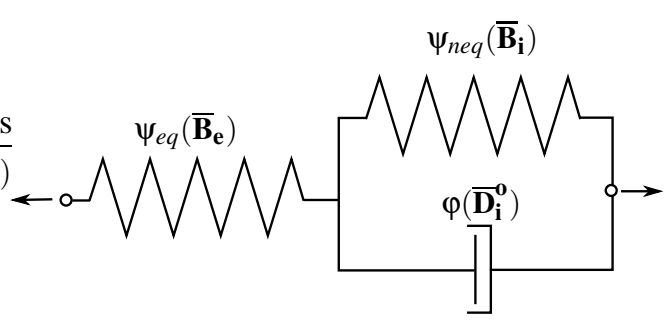

Figure 3: Poynting-Thomson model

The remainder inequality yields the definition of an internal stress-like variable, which is conjugate to an objective rate of the internal strain variable. This internal stress like variable is supposed to be derived from a pseudo-potential of dissipation $\varphi$, which is a convex positive function of the objective rate of the internal variable $\overline{\mathbf{D}}_{\mathbf{i}}^{\mathbf{0}}$ (with the constraint $\varphi(0)=0)$. It is then applied the normality principle to always satisfy the remainder inequality for all $\overline{\mathbf{D}}_{\mathbf{i}}^{\mathbf{0}}$ with $\operatorname{tr}\left(\overline{\mathbf{D}}_{\mathbf{i}}^{\mathbf{0}}\right)=$ 0 . The following complementary law can then be obtained:

$$
\frac{\partial \varphi}{\partial \overline{\mathbf{D}}_{\mathbf{i}}^{\mathbf{0}}}=\left(2 \rho_{0} J^{-1} \overline{\mathbf{V}}_{\mathbf{e}} \cdot \frac{\partial \psi_{n e q}}{\partial \overline{\mathbf{B}}_{\mathbf{e}}} \cdot \overline{\mathbf{V}}_{\mathbf{e}}\right)^{\mathrm{D}}
$$

Here, the simplest form of the pseudo dissipation potential $\varphi$ is assumed, i.e. a quadratic one:

$$
\varphi\left(\overline{\mathbf{D}}_{\mathbf{i}}^{\mathbf{o}}\right)=\frac{\eta}{2}\left(\overline{\mathbf{D}}_{\mathbf{i}}^{\mathbf{o}}: \overline{\mathbf{D}}_{\mathbf{i}}^{\mathbf{o}}\right)
$$

where $\eta$ is a viscosity parameter. Equation (13) gives

$$
\overline{\mathbf{D}}_{\mathbf{i}}^{\mathbf{o}}=\frac{2 \rho_{0}}{J \eta}\left(\overline{\mathbf{V}}_{\mathbf{e}} \cdot \frac{\partial \psi_{n e q}}{\partial \overline{\mathbf{B}}_{\mathbf{e}}} \cdot \overline{\mathbf{V}}_{\mathbf{e}}\right)^{\mathrm{D}}
$$

By substituting equation (15) into (8), the following evolution equation of the Zener model is obtained:

$$
\dot{\overline{\mathbf{B}}}_{\mathbf{e}}=\mathbf{L} \cdot \overline{\mathbf{B}}_{\mathbf{e}}+\overline{\mathbf{B}}_{\mathbf{e}} \cdot \mathbf{L}^{\mathrm{T}}-\frac{2}{3}(\mathbf{1}: \mathbf{L}) \overline{\mathbf{B}}_{\mathbf{e}}-\frac{2}{\eta} \boldsymbol{\sigma}_{n e q} \cdot \overline{\mathbf{B}}_{\mathbf{e}}
$$

\subsection{Poynting-Thomson model}

This model is presented in figure 3. It is defined with $\mathbf{X}=\overline{\mathbf{B}}_{\mathbf{e}}$ and $\mathbf{Y}=\overline{\mathbf{B}}_{\mathbf{i}}$. Based on (5), (7) and (8), the time derivative of the free energy is given by:

$$
\begin{aligned}
\dot{\psi}= & \frac{\partial \psi_{e q}}{\partial \overline{\mathbf{B}}_{\mathbf{e}}}:\left(\mathbf{L} \cdot \overline{\mathbf{B}}_{\mathbf{e}}+\overline{\mathbf{B}}_{\mathbf{e}} \cdot \mathbf{L}^{\mathrm{T}}-2 \overline{\mathbf{V}}_{\mathbf{e}} \cdot \overline{\mathbf{D}}_{\mathbf{i}}^{\mathbf{o}} \cdot \overline{\mathbf{V}}_{\mathbf{e}}-\frac{2}{3}(\mathbf{1}: \mathbf{D}) \overline{\mathbf{B}}_{\mathbf{e}}\right)+\frac{\partial \psi_{n e q}}{\partial \overline{\mathbf{B}}_{\mathbf{i}}}:\left(\overline{\mathbf{L}}_{\mathbf{i}} \cdot \overline{\mathbf{B}}_{\mathbf{i}}+\overline{\mathbf{B}}_{\mathbf{i}} \cdot \overline{\mathbf{L}}_{\mathbf{i}}^{\mathrm{T}}\right) \\
& +J \frac{\partial \psi_{v o l}}{\partial J}(\mathbf{1}: \mathbf{L})
\end{aligned}
$$


As with the previous model, a re-arrangement of the terms in the previous equation gives the following expression for the internal dissipation:

$$
\begin{aligned}
\mathcal{D}_{i n t}= & \left(\boldsymbol{\sigma}-\rho_{0} J^{-1}\left(2 \overline{\mathbf{B}}_{\mathbf{e}} \cdot \frac{\partial \psi_{e q}}{\partial \overline{\mathbf{B}}_{\mathbf{e}}}\right)^{\mathrm{D}}-\rho_{0}\left(\frac{\partial \psi_{v o l}}{\partial J} \mathbf{1}\right)\right): \mathbf{D} \\
& +\left(2 \rho_{0} J^{-1} \overline{\mathbf{V}}_{\mathbf{e}} \cdot \frac{\partial \psi_{e q}}{\partial \overline{\mathbf{B}}_{\mathbf{e}}} \cdot \overline{\mathbf{V}}_{\mathbf{e}}\right): \overline{\mathbf{D}}_{\mathbf{i}}^{\mathbf{o}} \\
& -\left(2 \rho_{0} J^{-1} \overline{\mathbf{B}}_{\mathbf{i}} \cdot \frac{\partial \psi_{\text {neq }}}{\partial \overline{\mathbf{B}}_{\mathbf{i}}}\right): \overline{\mathbf{D}}_{\mathbf{i}} \geq 0
\end{aligned}
$$

Using eq. (9) and the polar decomposition: $\overline{\mathbf{F}}_{\mathbf{e}}=\overline{\mathbf{V}}_{\mathbf{e}} \cdot \overline{\mathbf{R}}$, one can obtain the following expression:

$$
\overline{\mathbf{D}}_{\mathbf{i}}=\overline{\mathbf{F}}_{\mathbf{e}}^{\mathrm{T}} \cdot \overline{\mathbf{V}}_{\mathbf{e}}^{-1} \cdot \overline{\mathbf{D}}_{\mathbf{i}}^{\mathbf{o}} \cdot \overline{\mathbf{V}}_{\mathbf{e}}^{-1} \cdot \overline{\mathbf{F}}_{\mathbf{e}}
$$

A more practical form of the internal dissipation is thus obtained:

$$
\begin{aligned}
\mathcal{D}_{\text {int }}= & \left(\boldsymbol{\sigma}-\rho_{0} J^{-1}\left(2 \overline{\mathbf{B}}_{\mathbf{e}} \cdot \frac{\partial \psi_{e q}}{\partial \overline{\mathbf{B}}_{\mathbf{e}}}\right)^{\mathrm{D}}-\rho_{0}\left(\frac{\partial \psi_{v o l}}{\partial J} \mathbf{1}\right)\right): \mathbf{D} \\
& +\left(2 \rho_{0} J^{-1} \overline{\mathbf{V}}_{\mathbf{e}} \cdot \frac{\partial \psi_{e q}}{\partial \overline{\mathbf{B}}_{\mathbf{e}}} \cdot \overline{\mathbf{V}}_{\mathbf{e}}\right. \\
& \left.-2 \rho_{0} J^{-1} \overline{\mathbf{V}}_{\mathbf{e}}^{-1} \cdot \overline{\mathbf{F}}_{\mathbf{e}} \cdot \overline{\mathbf{B}}_{\mathbf{i}} \cdot \frac{\partial \psi_{n e q}}{\partial \overline{\mathbf{B}}_{\mathbf{i}}} \cdot \overline{\mathbf{F}}_{\mathbf{e}}^{\mathrm{T}} \overline{\mathbf{V}}_{\mathbf{e}}^{-1}\right): \overline{\mathbf{D}}_{\mathbf{i}}^{\mathbf{o}} \geq 0
\end{aligned}
$$

As done above, the constitutive equation is obtained for arbitrary choices of $\mathbf{D}$. The stress is a sum of a deviatoric hyperelastic and a spheric volumetric part:

$$
\boldsymbol{\sigma}=\overbrace{\left(2 \rho_{0} J^{-1} \overline{\mathbf{B}}_{\mathbf{e}} \cdot \frac{\partial \psi_{e q}}{\partial \overline{\mathbf{B}}_{\mathbf{e}}}\right)^{\mathrm{D}}}^{\boldsymbol{\sigma}_{e q}}+\overbrace{\rho_{0} \frac{\partial \psi_{v o l}}{\partial J} \mathbf{1}}^{\boldsymbol{\sigma}_{v o l}}
$$

For the remainder part, the existence of a pseudo potential of dissipation driving the internal stress-like variable is postulated. A more complex form can be obtained here than with the previous model:

$$
\frac{\partial \varphi}{\partial \overline{\mathbf{D}}_{\mathbf{i}}^{\mathbf{0}}}=2 \rho_{0} J^{-1}\left(\overline{\mathbf{V}}_{\mathbf{e}} \cdot \frac{\partial \psi_{e q}}{\partial \overline{\mathbf{B}}_{\mathbf{e}}} \cdot \overline{\mathbf{V}}_{\mathbf{e}}-2 \overline{\mathbf{V}}_{\mathbf{e}}^{-1} \cdot \overline{\mathbf{F}}_{\mathbf{e}} \cdot \overline{\mathbf{B}}_{\mathbf{i}} \cdot \frac{\partial \psi_{n e q}}{\partial \overline{\mathbf{B}}_{\mathbf{i}}} \cdot \overline{\mathbf{F}}_{\mathbf{e}}^{\mathrm{T}} \overline{\mathbf{V}}_{\mathbf{e}}^{-1}\right)^{\mathrm{D}}
$$

Assuming the free energy to be isotropic, and adopting the following notation for the derivative of $\psi_{\text {neq }}$ :

$$
\psi_{n e q, 1}=\frac{\partial \psi_{n e q}}{\partial I_{1}\left(\overline{\mathbf{B}}_{\mathbf{i}}\right)} \quad \psi_{n e q, 2}=\frac{\partial \psi_{n e q}}{\partial I_{2}\left(\overline{\mathbf{B}}_{\mathbf{i}}\right)}
$$

equation (22) becomes

$$
\overline{\mathbf{D}}_{\mathbf{i}}^{\mathbf{o}}=\frac{2 \rho_{0}}{J \eta}\left(\overline{\mathbf{B}}_{\mathbf{e}} \cdot \frac{\partial \psi_{e q}}{\partial \overline{\mathbf{B}}_{\mathbf{e}}}-2 \overline{\mathbf{V}}_{\mathbf{e}}^{-1} \cdot\left(\psi_{n e q, 1} \overline{\mathbf{B}}-\psi_{n e q, 2} \overline{\mathbf{B}}_{\mathbf{e}} \cdot \overline{\mathbf{B}}^{-1} \cdot \overline{\mathbf{B}}_{\mathbf{e}}\right) \cdot \overline{\mathbf{V}}_{\mathbf{e}}^{-1}\right)^{\mathrm{D}}
$$

By substituting equation (24) into (8), we obtain the following evolution equation for the internal variable of a Poynting Thomson model:

$$
\begin{aligned}
\dot{\overline{\mathbf{B}}}_{\mathbf{e}}= & \mathbf{L} \cdot \overline{\mathbf{B}}_{\mathbf{e}}+\overline{\mathbf{B}}_{\mathbf{e}} \cdot \mathbf{L}^{\mathrm{T}}-\frac{2}{3}(\mathbf{1}: \mathbf{L}) \overline{\mathbf{B}}_{\mathbf{e}}-\frac{2}{\eta} \boldsymbol{\sigma}_{e q} \cdot \overline{\mathbf{B}}_{\mathbf{e}} \\
& +\frac{4}{J \eta} \psi_{n e q, 1}\left(\overline{\mathbf{B}}-\frac{1}{3}\left(\overline{\mathbf{B}}: \overline{\mathbf{B}}_{\mathbf{e}}^{-1}\right) \overline{\mathbf{B}}_{\mathbf{e}}\right) \\
& +\frac{4}{J \eta} \psi_{n e q, 2}\left(\overline{\mathbf{B}}_{\mathbf{e}} \cdot \overline{\mathbf{B}}^{-1} \cdot \overline{\mathbf{B}}_{\mathbf{e}}-\frac{1}{3}\left(\overline{\mathbf{B}}^{-1}: \overline{\mathbf{B}}_{\mathbf{e}}\right) \overline{\mathbf{B}}_{\mathbf{e}}\right)
\end{aligned}
$$




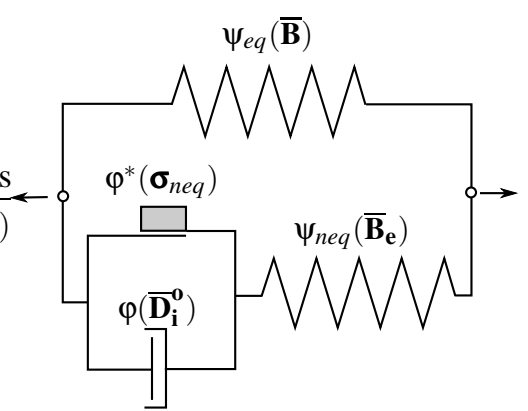

Figure 4: A Visco-plastic model

\subsection{Generalized Bingham model}

The visco-plastic model, presented in figure 4, consists of a Bingham viscoplastic element put in parallel with an elastic one. In this model, we have $\mathbf{X}=\overline{\mathbf{B}}$ and $\mathbf{Y}=\overline{\mathbf{B}}_{\mathbf{e}}$. The same constitutive equation is obtained as in the case of the first viscoelastic model :

$$
\boldsymbol{\sigma}=\overbrace{\left(2 \rho_{0} J^{-1} \overline{\mathbf{B}} \cdot \frac{\partial \psi_{e q}}{\partial \overline{\mathbf{B}}}\right)^{\mathrm{D}}}^{\boldsymbol{\sigma}_{e q}}+\overbrace{\left(2 \rho_{0} J^{-1} \overline{\mathbf{B}}_{\mathbf{e}} \cdot \frac{\partial \psi_{\text {neq }}}{\partial \overline{\mathbf{B}}_{\mathbf{e}}}\right)^{\mathrm{D}}}^{\boldsymbol{\sigma}_{\text {neq }}}+\overbrace{\rho_{0} \frac{\partial \psi_{\text {vol }}}{\partial J} \mathbf{1}}^{\boldsymbol{\sigma}_{\text {vol }}}
$$

For the remainder part of the internal dissipation, we take the following Legendre-Fenchel transformation of the pseudo-potential of dissipation:

$$
\varphi^{*}=\frac{\left\langle f\left(\boldsymbol{\sigma}_{\text {neq }}\right)\right\rangle}{\eta} \quad \text { with } \quad f\left(\boldsymbol{\sigma}_{\text {neq }}\right)=\left\|\boldsymbol{\sigma}_{\text {neq }}\right\|-\chi
$$

where $\eta$ is a viscosity parameter, $\left\langle.>\right.$ are the Mac-Cauley brackets ${ }^{1}$, and the function $f$ is a Yield stress function that defines the elastic domain $\mathbb{E}$ :

$$
\mathbb{E}=\left\{\boldsymbol{\sigma}_{\text {neq }} \mid f\left(\boldsymbol{\sigma}_{\text {neq }}\right) \leq 0\right\}
$$

The application of the normality principle leads to the following expression:

$$
\overline{\mathbf{D}}_{\mathbf{i}}^{\mathbf{o}}=\frac{\partial \varphi^{*}}{\partial \boldsymbol{\sigma}_{n e q}}=\frac{\left\langle f\left(\boldsymbol{\sigma}_{n e q}\right)\right\rangle}{\eta} \frac{\boldsymbol{\sigma}_{n e q}}{\left\|\boldsymbol{\sigma}_{n e q}\right\|}
$$

By subsitituting equation (29) into (8) and using the isotropy of the free energy, the following flow rule is obtained

$$
\dot{\mathbf{B}}_{\mathbf{e}}=\mathbf{L} \cdot \overline{\mathbf{B}}_{\mathbf{e}}+\overline{\mathbf{B}}_{\mathbf{e}} \cdot \mathbf{L}^{\mathrm{T}}-\frac{2}{3}(\mathbf{1}: \mathbf{L}) \overline{\mathbf{B}}_{\mathbf{e}}-2 \frac{\left\langle f\left(\boldsymbol{\sigma}_{n e q}\right)\right\rangle}{\eta} \frac{\boldsymbol{\sigma}_{n e q}}{\left\|\boldsymbol{\sigma}_{n e q}\right\|} \cdot \overline{\mathbf{B}}_{\mathbf{e}}
$$

\section{Variational Formulation}

In this paper, the so-called perturbed Lagrangian variational form is adopted. This variational form is a twofield formulation accounting satisfactorily for nearly-incompressible behavior. In this formulation, the Lagrangian parameter has a physical meaning of the hydrostatic pressure thanks to the decomposition of $\mathbf{F}$ into isochoric and volumetric parts (see [19] for more details). Introducing the couple (u; $p$ ) denoting the kinematics and the hydrostatic

\footnotetext{
${ }^{1}$ Operator $<.>$ is defined by $<f>=f$ if $f \geq 0$ and $<f>=0$ if $f<0$
} 
pressure, the solution of the equilibrium problem has to cancel the following integral form for all the test functions $\delta \mathbf{v}$ and $\delta p$ chosen in the same spaces as those of the trial functions $\mathbf{u}$ and $p$ respectively ${ }^{2}$ :

$$
\left\{\begin{array}{l}
\int_{\Omega_{0}} \Pi: \nabla \delta v d \Omega-\int_{\Omega_{0}} \delta v \cdot \mathbf{f}_{\mathbf{v o l}} d \Omega-\int_{\delta \Omega} \delta v \cdot \mathbf{f}_{\text {surf }} d S=0 \\
\int_{\Omega_{0}}\left(\rho_{0} \frac{\partial \psi_{v o l}}{\partial J}-p\right) \delta q d \Omega=0
\end{array}\right.
$$

where $\Pi$ is the first Piola Kirchoff stress. To proceed further, a classical quadratic volumetric potential is adopted, which gives the following linear pressure law:

$$
\rho_{0} \psi_{v o l}=\frac{k}{2}(J-1)^{2} \quad \Rightarrow \quad p=k(J-1)
$$

where $k$ is the compressibility modulus. Equations (31) become

$$
\left\{\begin{array}{l}
\int_{\Omega_{0}}\left(\boldsymbol{\Pi}_{i s o}(\mathbf{u})+p J \mathbf{F}^{-\mathrm{T}}\right): \nabla \delta v d \Omega-\int_{\Omega_{0}} \delta v \cdot \mathbf{f}_{\mathbf{v o l}} d \Omega-\int_{\delta \Omega} \delta v \cdot \mathbf{f}_{\text {surf }} d S=0 \\
\int_{\Omega_{0}}\left(J(\mathbf{u})-1-\frac{p}{k}\right) \delta q d \Omega=0
\end{array}\right.
$$

It can be seen from the above equations that $1 / k$ act as a perturbation parameter enforcing incompressibility. The isochoric stress part of the first Piola-Kirchoff stress is computed by a simple transport of the Cauchy stress, so that

$$
\begin{aligned}
& \boldsymbol{\Pi}_{i s o}=J \boldsymbol{\sigma}_{i s o} \cdot \mathbf{F}^{-\mathrm{T}}=J\left(\boldsymbol{\sigma}_{e q}+\boldsymbol{\sigma}_{n e q}\right) \cdot \mathbf{F}^{-\mathrm{T}} \quad \text { Zener and Visco-plastic models } \\
& \boldsymbol{\Pi}_{i s o}=J \boldsymbol{\sigma}_{i s o} \cdot \mathbf{F}^{-\mathrm{T}}=J\left(\boldsymbol{\sigma}_{e q}\right) \cdot \mathbf{F}^{-\mathrm{T}} \quad \text { Poynting Thomson model }
\end{aligned}
$$

For more precisions on the variational formulation and the finite elements implementation in the context of mixed formulations for incompressible media submitted to large strain, we refer to the work of $[20,21,22,23]$ and references therein.

\section{Numerical Integration of the Flow Rule}

\subsection{Scalar Backward Euler with Time-Stepping (BETS)}

A local incremental process within the Newton-Raphson scheme is used to solve the equilibrium equations. In the time interval $\left[t_{k}, t_{k+1}\right]$ all is given at time $t_{k}$ and the Newton-Raphson scheme gives a prediction of $\mathbf{F}$ at time $t_{k+1}$. We therefore look for the internal variables at time $t_{k+1}$. In what follows $\mathbf{F}$ is assumed to be a linear function of time in the interval $\left[t_{k}, t_{k+1}\right]$. This implies $\mathbf{F}$ is constant (by time interval), and defined by:

$$
\dot{\mathbf{F}}=\frac{\Delta \mathbf{F}}{\Delta t}=\frac{\mathbf{F}_{\left.\right|_{k+1}}-\mathbf{F}_{\mid k}}{t_{k+1}-t_{k}}
$$

\footnotetext{
${ }^{2} \mathbf{f}_{v o l}$ and $\mathbf{f}_{\text {surf }}$ are the volumetric external forces and the surface forces respectively.
} 
In line with $[1,7]$, among others, one can observe that the flow rule takes a generic tensorial form, in which pure elastic and inelastic parts can be distinguished ${ }^{3}$ :

$$
\begin{aligned}
& \left\{\begin{array}{l}
\dot{\overline{\mathbf{B}}}_{\mathbf{e}}=\mathcal{G}\left(\overline{\mathbf{B}}_{\mathbf{e}}, \mathbf{L}, \overline{\mathbf{B}}\right)=\underbrace{\mathcal{H}\left(\overline{\mathbf{B}}_{\mathbf{e}}, \mathbf{L}\right)}_{\text {elastic }}+\underbrace{I\left(\overline{\mathbf{B}}_{\mathbf{e}}, \overline{\mathbf{B}}\right)}_{\text {inelastic }} \quad t \in\left[t_{k}, t_{k+1}\right] \\
\overline{\mathbf{B}}_{\mathbf{e}}\left(t_{k}\right)=\left.\overline{\mathbf{B}}_{\mathbf{e}}\right|_{k}
\end{array}\right. \\
& \text { with } \mathcal{H}\left(\overline{\mathbf{B}}_{\mathbf{e}}, \mathbf{L}\right)=\mathbf{L} \cdot \overline{\mathbf{B}}_{\mathbf{e}}+\overline{\mathbf{B}}_{\mathbf{e}} \cdot \mathbf{L}^{\mathrm{T}}-\frac{2}{3}(\mathbf{1}: \mathbf{L}) \overline{\mathbf{B}}_{\mathbf{e}} \\
& \qquad \begin{array}{l}
-\frac{2}{\eta} \boldsymbol{\sigma}_{\text {neq }} \cdot \overline{\mathbf{B}}_{\mathbf{e}} \quad \text { for Zener } \\
-\frac{2}{\eta} \boldsymbol{\sigma}_{e q} \cdot \overline{\mathbf{B}}_{\mathbf{e}}+\frac{4}{J \eta} \psi_{\text {neq }, 1}\left(\overline{\mathbf{B}}-\frac{1}{3}\left(\overline{\mathbf{B}}: \overline{\mathbf{B}}_{\mathbf{e}}{ }^{-1}\right) \overline{\mathbf{B}}_{\mathbf{e}}\right)+ \\
\frac{4}{J \eta} \psi_{\text {neq }, 2}\left(\overline{\mathbf{B}}_{\mathbf{e}} \cdot \overline{\mathbf{B}}^{-1} \cdot \overline{\mathbf{B}}_{\mathbf{e}}-\frac{1}{3}\left(\overline{\mathbf{B}}^{-1}: \overline{\mathbf{B}}_{\mathbf{e}}\right) \overline{\mathbf{B}}_{\mathbf{e}}\right) \quad \text { for Poynthing-Thomson } \\
-2 \frac{\left\langle f\left(\boldsymbol{\sigma}_{\text {neq }}\right)\right\rangle}{\eta} \frac{\boldsymbol{\sigma}_{\text {neq }}}{\left\|\boldsymbol{\sigma}_{\text {neq }}\right\|} \cdot \overline{\mathbf{B}}_{\mathbf{e}} \quad \text { for Bingham }
\end{array}
\end{aligned}
$$

Splitting the differential system is particularly interesting to introduce a new intermediate configuration. Taking a substep defined by a sub-interval ${ }^{4}\left[t_{n}, t_{n+1}\right]$ of size $h$, all the state variables are evaluated at the time $t_{n}$ and the intermediate trial elastic state at time $t_{n+1}$ is defined from

$$
\overline{\mathbf{F}}_{\mathbf{e}}^{\star}=\overline{\mathbf{F}}_{\mid n+1} \cdot \overline{\mathbf{F}}_{\mathbf{i} \mid n}^{-1}
$$

The trial left Cauchy-Green tensor is then defined by

$$
\overline{\mathbf{B}}_{\mathbf{e}}^{\star}=\overline{\mathbf{F}}_{\mid n+1} \cdot \overline{\mathbf{F}}_{\mid n}^{-1} \cdot \overline{\mathbf{B}}_{\mathbf{e} \mid n} \cdot \overline{\mathbf{F}}_{\mid n}^{-\mathrm{T}} \cdot \overline{\mathbf{F}}_{\mid n+1}{ }^{\mathrm{T}}
$$

This trial state is the solution of equation (36) if we set $I\left(\overline{\mathbf{B}}_{\mathbf{e}}, \overline{\mathbf{B}}\right)=0$, which corresponds to a pure elastic solution. As in exponential mapping and radial return mapping schemes, a predictive/corrective algorithm is considered, in which the predictive solution is the trial state. Therefore, the corrective step consists in solving the following modified differential tensorial system (we set $\mathbf{L}=0$ and so $\mathcal{H}\left(\overline{\mathbf{B}}_{\mathbf{e}}, \mathbf{L}\right)=0$ ):

$$
\left\{\begin{array}{l}
\dot{\overline{\mathbf{B}}}_{\mathbf{e}}=I\left(\overline{\mathbf{B}}_{\mathbf{e}}, \overline{\mathbf{B}}\right) \quad t \in\left[t_{n}, t_{n+1}\right] \\
\overline{\mathbf{B}}_{\mathbf{e}}\left(t_{n}\right)=\overline{\mathbf{B}}_{\mathbf{e}}^{\star}
\end{array}\right.
$$

To proceed further, it is assumed that the solution can be decomposed as follows:

$$
\overline{\mathbf{B}}_{\mathbf{e}}(t)=b(t) \mathbf{N}+s(t) \mathbf{1}
$$

where $\mathbf{N}$ denotes a normalized deviatoric strain tensor with the following basic properties:

$$
(\mathbf{N}: \mathbf{N})=1 \text { and }(\mathbf{N}: \mathbf{1})=0 \quad \Rightarrow \quad(\dot{\mathbf{N}}: \mathbf{N})=0 \text { and }(\dot{\mathbf{N}}: \mathbf{1})=0
$$

This tensor can be taken to give the flow direction, $b(t)$ and $s(t)$ are the flow amplitudes defined by

$$
b(t)=\overline{\mathbf{B}}_{\mathbf{e}}: \mathbf{N} \quad s(t)=\frac{1}{3} \overline{\mathbf{B}}_{\mathbf{e}}: \mathbf{1}
$$

The main assumption involved in the proposed algorithm is based on the following choice of the flow direction $\mathbf{N}$ :

$$
\mathbf{N}=\frac{\overline{\mathbf{B}}_{\mathbf{e}}{ }^{{ }^{\mathrm{D}}}+h I\left(\overline{\mathbf{B}}_{\mathbf{e}}{ }^{\star}, \overline{\mathbf{B}}_{\mid n+1}\right)^{\mathrm{D}}}{\left\|\overline{\mathbf{B}}_{\mathbf{e}}{ }^{{ }^{\mathrm{D}}}+h I\left(\overline{\mathbf{B}}_{\mathbf{e}}{ }^{\star}, \overline{\mathbf{B}}_{\mid n+1}\right)^{\mathrm{D} \|}\right\|}
$$

\footnotetext{
${ }^{3}$ For the sake of simplicity the dependence on time of the tensorial quantity is not explicitly written

${ }^{4} \mathrm{~A}$ sub-step is a time partition of the time interval $\left[t_{k}, t_{k+1}\right]$
} 
This choice make it possible to take into account both the time evolution ${ }^{5}$ of $\mathbf{N}$ and its dependence on the nature of the flow rule.

The time derivative of $\overline{\mathbf{B}}_{\mathbf{e}}$ is then:

$$
\dot{\overline{\mathbf{B}}}_{\mathbf{e}}=\dot{b} \mathbf{N}+b \dot{\mathbf{N}}+\dot{s} \mathbf{1}
$$

Introducing (40) and (44) into (39), the tensorial flow rule can be reduced to a scalar differential system of 2 equations with the two unknowns $\{s, b\}$. This system is obtained directly by using the properties of $\mathbf{N}$ in eq. (41), i.e. using the projections $\dot{\overline{\mathbf{B}}}_{\mathbf{e}}: \mathbf{1}$ and $\dot{\overline{\mathbf{B}}}_{\mathbf{e}}: \mathbf{N}$. Appendix A sums up the details of the calculations in the case of each rheological model. A vector $\mathbf{y}$ is then introduced, that contains the two unknowns $\{s, b\}$. The differential system can be written as follows:

$$
\left\{\begin{array}{l}
\dot{\mathbf{y}}=\mathcal{N}(\mathbf{y}, \mathbf{N}, \overline{\mathbf{B}}) \\
\mathbf{y}_{n}=\left\{s^{\star}, b^{\star}\right\}=\left\{\frac{\overline{\mathbf{B}}_{\mathbf{e}}^{\star}: \mathbf{1}}{3}, \overline{\mathbf{B}}_{\mathbf{e}}^{\star}: \mathbf{N}\right\}
\end{array}\right.
$$

Where $\mathcal{N}$ is the vectorial form of the inelastic part of the flow rule $I$. The ordinary differential system is non-linear and with non-constant coefficients, since $\mathbf{N}$ and $\overline{\mathbf{B}}$ depend on time. This system is solved with a Backward-Euler scheme:

$$
\mathbf{y}_{n+1}=\mathbf{y}_{n}+h \mathcal{N}\left(\mathbf{y}_{n+1}, \mathbf{N}, \overline{\mathbf{B}}_{\left.\right|_{n+1}}\right)
$$

This process leads to the resolution of a non-linear system, using a local Newton scheme.

The sub-step size, $h$, is controlled by an auto-adaptive scheme, taking the following local error:

$$
\begin{aligned}
& \text { LER }=\left\|\dot{\overline{\mathbf{B}}}_{\left.\mathbf{e}\right|_{\mathbf{n}+1}}-\dot{\overline{\mathbf{B}}}_{\left.\mathbf{e}\right|_{\mathbf{n}+\mathbf{1}}}^{(1 / 2)}\right\|=\frac{1}{h}\left\|\frac{1}{2} \overline{\mathbf{B}}_{\left.\mathbf{e}\right|_{n+1}}-2 \overline{\mathbf{B}}_{\left.\mathbf{e}\right|_{n+\frac{1}{2}}}+\frac{3}{2} \overline{\mathbf{B}}_{\left.\mathbf{e}\right|_{n} \|}\right\| \\
& \text { with } \quad \dot{\overline{\mathbf{B}}}_{\left.\mathbf{e}\right|_{\mathbf{n}+\mathbf{1}}}=\frac{\overline{\mathbf{B}}_{\left.\mathbf{e}\right|_{n+1}}-\overline{\mathbf{B}}_{\left.\mathbf{e}\right|_{n}}}{h} \\
& \text { and } \quad \dot{\overline{\mathbf{B}}}_{\left.\mathbf{e}\right|_{\mathbf{n}+1}}^{(1 / 2)}=\frac{1}{h}\left(\frac{3}{2} \overline{\mathbf{B}}_{\left.\mathbf{e}\right|_{n+1}}-2 \overline{\mathbf{B}}_{\left.\mathbf{e}\right|_{n+\frac{1}{2}}}+\frac{1}{2} \overline{\mathbf{B}}_{\left.\mathbf{e}\right|_{n}}\right)
\end{aligned}
$$

This error requires a mid step evaluation at $t_{n+\frac{1}{2}}$. Therefore, at each Gauss point, the local Newton scheme is used twice to evaluate $\overline{\mathbf{B}}_{\left.\mathbf{e}\right|_{n+\frac{1}{2}}}$ and $\overline{\mathbf{B}}_{\left.\mathbf{e}\right|_{n+1}}$. Whenever this local error estimate satisfies $L E R<T O L$, the current sub-step is accepted and the new sub-step size is computed from

$$
h_{\text {new }}=0.9 \max \left(r_{\text {min }}, \min \left(r_{\text {max }},\left(\frac{T O L}{L E R}\right)^{1 / 3}\right)\right) h_{\text {old }}
$$

where TOL is the desired error tolerance, and $r_{\min }$ and $r_{\max }$ are the minimum and maximum bounds of the sub-step size variation preventing to small or to large $h$ values. If the local error is greater than the error tolerance, the sub-step is rejected and re-tested with a smaller sub-step size (given by eq. (48)). However, from a numerical point of view, it can be experimented some cases where $r_{\text {min }}$ is reached. In this cases, the global-step size is reduced and the integration process is retried. This heuristic scheme may conduced to situations where the auto-adaptive scheme is very costly. The choice of $r_{\min }, r_{\max }$ and TOL must be done with care from numerical experiments.

\subsection{Linearization of the scalar Backward Euler with Time-Stepping (LBETS)}

Starting with the assumption (40), a series expansion of the unknown is performed around $\left\{s^{\star}, b^{\star}\right\}$ so that

$$
s(t)=s^{\star}+\delta s(t) \quad b(t)=b^{\star}+\delta b(t)
$$

\footnotetext{
${ }^{5}$ as $\overline{\mathbf{B}}_{\mathbf{e}}{ }^{{ }^{\mathrm{D}}}, \overline{\mathbf{B}}$ depend on time.
} 
By introducing (49) into the vectorial flow rule $\mathcal{N}$ and keeping only first order terms, the following approximated differential system can be obtained

$$
\left\{\begin{array}{c}
\dot{\delta s} \\
\dot{\delta b}
\end{array}\right\}=A(t)\left\{\begin{array}{l}
\delta s \\
\delta b
\end{array}\right\}+C(t)
$$

with the following initial conditions :

$$
\delta s\left(t_{n}\right)=\delta b\left(t_{n}\right)=0
$$

As we are looking for exponential solutions, the system (50) is solved using a semi-explicit scheme in which $A(t)$ and $C(t)$ are fixed at time $t_{n}$. With this approach the vectorial differential system has an analytical solution, which can be computed from:

$$
\left\{\begin{array}{l}
\delta s\left(t_{n+1}\right) \\
\delta b\left(t_{n+1}\right)
\end{array}\right\}=\left(\int_{t_{n}}^{t_{n+1}} e^{A\left(t_{n+1}-s\right)} d s\right) C=\left(e^{A \Delta t}-I\right) A^{-1} C
$$

where $I$ is the identity matrix, and $e^{A}$ is the exponential matrix of $A$. By taking into account the time dependence of the operator $A$ and $C$, a semi analytical solution can be exhibited that also takes the form of an exponential with Magnus series. The authors will present this specific integration scheme in a paper to appear [24].

The aforementioned exponential matrix is computed using a squalling and squaring method with Padé approximants (for other strategies, see (author?) [25], who compared of 19 different methods). The main idea is to use the property $e^{A}=\left(e^{A / 2^{j}}\right)^{2^{j}}$ and to compute $e^{A / 2^{j}}$ with Padé approximants:

$$
e^{A / 2^{j}} \simeq\left[N_{q}\left(-A / 2^{j}\right)\right]^{-1} N_{q}\left(A / 2^{j}\right)
$$

where

$$
N_{q}\left(A / 2^{j}\right)=\sum_{i=0}^{q} \frac{(2 q-i) ! q !}{2 q ! i !(q-i) !}\left(A / 2^{j}\right)^{i}
$$

and $j$ is chosen such as $\|A\| \leq 2^{j-1}$ and $q=2$.

The same time stepping strategy is used here as with the previous integrator, adopting the same local error.

\subsection{Tangent moduli}

The specific tensorial operators used in this section are defined in Annex B. Linearizing equations (33), with a view to implementing a Newton-Raphson algorithm, leads to calculating the tangent moduli:

$$
\frac{\partial \boldsymbol{\Pi}_{i s o}}{\partial \mathbf{F}}=\tau_{i s o} \bullet \frac{\partial \mathbf{F}^{-\mathrm{T}}}{\partial \mathbf{F}}+\frac{\partial \tau_{i s o}}{\partial \mathbf{F}}\left(2 \mathbf{F}^{-\mathrm{T}}\right.
$$

The Kirchoff stress $\tau_{i s o}=J \boldsymbol{\sigma}_{i s o}$ is introduced into the previous relation. The main difficulty resides in obtaining the derivative of $\tau_{i s o}$ with respect to $\mathbf{F}$. Using $\tau_{i s o}=\tau_{e q}(\mathbf{X})+\tau_{\text {neq }}(\mathbf{Y})$, where $\mathbf{X}$ and $\mathbf{Y}$ are $\overline{\mathbf{B}}_{\mathbf{e}}$ or $\overline{\mathbf{B}}$, the following expression is obtained:

$$
\frac{\partial \tau_{i s o}}{\partial \mathbf{F}}=\frac{\partial \tau_{e q}}{\partial \mathbf{X}}: \frac{\partial \mathbf{X}}{\partial \mathbf{F}}+\frac{\partial \tau_{n e q}}{\partial \mathbf{Y}}: \frac{\partial \mathbf{Y}}{\partial \mathbf{F}}
$$

Calculating $\partial \tau_{e q} / \partial \mathbf{X}, \partial \tau_{n e q} / \partial \mathbf{Y}$ or $\partial \overline{\mathbf{B}} / \partial \mathbf{F}$ does not involve any specific difficulties and will not be detailed in this paper: see $[26,27]$ and the references therein for further details. Therefore, it still remains to calculate $\partial \overline{\mathbf{B}}_{\mathbf{e}} / \partial \mathbf{F}$.

Starting with a backward-Euler scheme, one has:

$$
\overline{\mathbf{B}}_{\left.\mathbf{e}\right|_{k+1}}=\overline{\mathbf{B}}_{\mathbf{e}}^{\star}-\Delta t I\left(\overline{\mathbf{B}}_{\left.\mathbf{e}\right|_{k+1}}, \overline{\mathbf{B}}_{\left.\right|_{k+1}}\right)
$$

where $\Delta t=t_{k+1}-t_{k}$. A direct derivation gives:

$$
\left.\frac{\partial \overline{\mathbf{B}}_{\mathbf{e}}}{\partial \mathbf{F}}\right|_{k+1}=\left.\frac{\partial \overline{\mathbf{B}}_{\mathbf{e}}^{\star}}{\partial \mathbf{F}}\right|_{k+1}-\left.\Delta t \frac{\partial I\left(\left.\overline{\mathbf{B}}_{\mathbf{e}}\right|_{k+1}, \overline{\mathbf{B}}_{\left.\right|_{k+1}}\right)}{\partial \mathbf{F}}\right|_{k+1}
$$


with

$$
\begin{aligned}
\left.\frac{\partial \overline{\mathbf{B}}_{\mathbf{e}}^{\star}}{\partial \mathbf{F}}\right|_{k+1}= & \frac{\partial \overline{\mathbf{F}}}{\partial \mathbf{F}}(2)\left(\left.\overline{\mathbf{F}}_{\left.\right|_{k}}^{-1} \cdot \overline{\mathbf{B}}_{\mathbf{e}}\right|_{k} \cdot \overline{\mathbf{F}}_{\left.\right|_{k}}^{-\mathrm{T}} \cdot \overline{\mathbf{F}}_{\left.\right|_{k+1}}^{\mathrm{T}}\right) \\
& +\left(\overline{\mathbf{F}}_{\left.\right|_{k+1}} \cdot \overline{\mathbf{F}}_{\left.\right|_{k}}^{-1} \cdot \overline{\mathbf{B}}_{\mathbf{e}_{k}} \cdot \overline{\mathbf{F}}_{\left.\right|_{k}}^{-\mathrm{T}}\right) \bullet \frac{\partial \overline{\mathbf{F}}^{\mathrm{T}}}{\partial \mathbf{F}}
\end{aligned}
$$

and the derivative of $\overline{\mathbf{F}}$, which are defined by

$$
\begin{aligned}
& \frac{\partial \overline{\mathbf{F}}}{\partial \mathbf{F}}=J^{-1 / 3}\left(\mathbb{I}-\frac{1}{3} \mathbf{F} \otimes \mathbf{F}^{-\mathrm{T}}\right) \\
& \frac{\partial \overline{\mathbf{F}}^{\mathrm{T}}}{\partial \mathbf{F}}=J^{-1 / 3}\left(\mathbf{1} \otimes \mathbf{1}-\frac{1}{3} \mathbf{F}^{\mathrm{T}} \otimes \mathbf{F}^{-\mathrm{T}}\right)
\end{aligned}
$$

The derivative of $I$ is specific to each rheological model and does not involve any specific difficulties. This gives:

$$
\frac{\partial I\left(\left.\overline{\mathbf{B}}_{\mathbf{e}}\right|_{k+1}, \overline{\mathbf{B}}_{k+1}\right)}{\partial \mathbf{F}}=\frac{\partial I\left(\overline{\mathbf{B}}_{\mathbf{e}}, \overline{\mathbf{B}}\right)}{\partial \overline{\mathbf{B}}_{\mathbf{e}}}:\left.\frac{\partial \overline{\mathbf{B}}_{\mathbf{e}}}{\partial \mathbf{F}}\right|_{k+1}+\frac{\partial I\left(\overline{\mathbf{B}}_{\mathbf{e}}, \overline{\mathbf{B}}\right)}{\partial \overline{\mathbf{B}}}:\left.\frac{\partial \overline{\mathbf{B}}}{\partial \mathbf{F}}\right|_{k+1}
$$

Finally $\partial \overline{\mathbf{B}}_{\mathbf{e}} / \partial \mathbf{F}$ can be obtained from the resolution of a linear system.

In the case of the present linearized scheme, the derivative of $\overline{\mathbf{B}}_{\mathbf{e}}$ is calculated as follows

$$
\frac{\partial \overline{\mathbf{B}}_{\mathbf{e}}}{\partial \mathbf{F}}=\mathbf{N}^{\star} \otimes \frac{\partial \delta b}{\partial \mathbf{F}}+\mathbf{1} \otimes \frac{\partial \delta s}{\partial \mathbf{F}}+\delta b \frac{\partial \mathbf{N}^{\star}}{\partial \mathbf{F}}+\frac{\partial \overline{\mathbf{B}}_{\mathbf{e}}^{\star}}{\partial \mathbf{F}}
$$

The terms $\partial \delta b / \partial \mathbf{F}$ and $\partial \delta s / \partial \mathbf{F}$ are obtained from the linear differential system of equation (50):

$$
\left\{\begin{array}{c}
\frac{\partial \dot{\delta} s}{\partial \mathbf{F}_{i j}} \\
\frac{\partial \dot{\delta} b}{\partial \mathbf{F}_{i j}}
\end{array}\right\}=A(t)\left\{\begin{array}{l}
\frac{\partial \delta s}{\partial \mathbf{F}_{i j}} \\
\frac{\partial \delta b}{\partial \mathbf{F}_{i j}}
\end{array}\right\}+\frac{\partial C}{\partial \mathbf{F}_{i j}}+\frac{\partial A}{\partial \mathbf{F}_{i j}}\left\{\begin{array}{c}
\delta s \\
\delta b
\end{array}\right\}
$$

The solution of the above differential system is obtained using the the same expression as in (52), which also involves an exponential matrix evaluation.

\section{Examples}

The folling examples are given to show the reliability and the numerical performances of the proposed schemes. These schemes will be compared with a 4th order Runge-Kutta scheme with time stepping (RKF), which is breifly presented in Annex C. The finite elements code ZeBuLoN (see [28]) was used to implement these schemes.

The following free energies were chosen for the rheological models:

- for the Zener and Generalized Bingham models:

$$
\left\{\begin{array}{l}
\psi_{e q}(\overline{\mathbf{B}})=C_{1}\left(I_{1}(\overline{\mathbf{B}})-3\right)+C_{2}\left(I_{2}(\overline{\mathbf{B}})-3\right)+C_{3}\left(I_{1}(\overline{\mathbf{B}})-3\right)^{3} \\
\psi_{\text {neq }}\left(\overline{\mathbf{B}}_{\mathbf{e}}\right)=G\left(I_{1}\left(\overline{\mathbf{B}}_{\mathbf{e}}\right)-3\right)
\end{array}\right.
$$

\begin{tabular}{lllllll}
\hline model & $C_{1}(\mathrm{MPa})$ & $C_{2}(\mathrm{MPa})$ & $C_{3}(\mathrm{MPa})$ & $G(\mathrm{MPa})$ & $\eta(\mathrm{MPa} . \mathrm{s})$ & $\chi(\mathrm{MPa})$ \\
\hline Zener & $7.08 \mathrm{e}-02$ & $-8.42 \mathrm{e}-02$ & $5.27 \mathrm{e}-02$ & $6.14 \mathrm{e}-01$ & $5.06 \mathrm{e}-01$ & \\
Poynting-Thomson & $6.16 \mathrm{e}-01$ & $-4.52 \mathrm{e}-02$ & 2.01 & 1.78 & $4.89 \mathrm{e}-01$ & \\
Visco-plastic & $2.68 \mathrm{e}-01$ & $2.37 \mathrm{e}-01$ & $2.10 \mathrm{e}-02$ & $8.25 \mathrm{e}-01$ & $1.29 \mathrm{e}-01$ & $1.33 \mathrm{e}-01$ \\
\hline
\end{tabular}

Table 1: Material parameters 


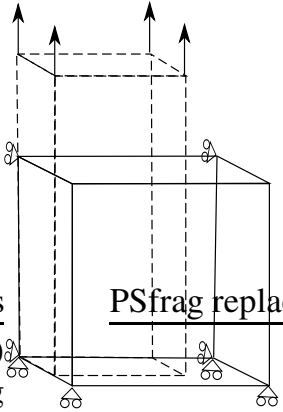

(a) boundary conditions

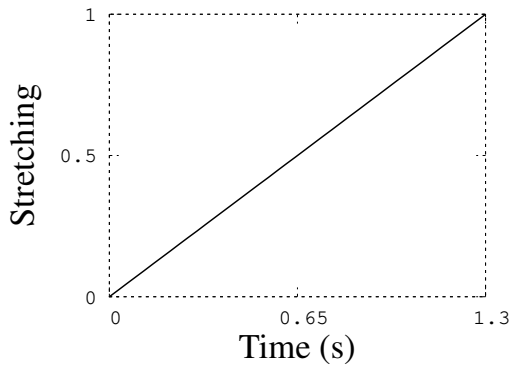

(b) loading path

Figure 5: Tensile relaxation test

- and for the Poynting-Thomson model:

$$
\left\{\begin{array}{l}
\psi_{e q}\left(\overline{\mathbf{B}}_{\mathbf{e}}\right)=C_{1}\left(I_{1}\left(\overline{\mathbf{B}}_{\mathbf{e}}\right)-3\right)+C_{2}\left(I_{2}\left(\overline{\mathbf{B}}_{\mathbf{e}}\right)-3\right)+C_{3}\left(I_{1}\left(\overline{\mathbf{B}}_{\mathbf{e}}\right)-3\right)^{3} \\
\psi_{\text {neq }}\left(\overline{\mathbf{B}}_{\mathbf{i}}\right)=G\left(I_{1}\left(\overline{\mathbf{B}}_{\mathbf{i}}\right)-3\right)
\end{array}\right.
$$

The material parameters are fitted to the experimental data (traction relaxation and cyclic shear tests from [29]). Table 1 sums up the material parameters identified. A value of $k=1500 \mathrm{MPa}$ is adopted for the compressibility modulus.

\subsection{Uniform traction}

In this test, a unit cube of rubber is subjected to a uniform stretching of $\lambda=2$. Due to the symmetry of the boundary conditions, only a quarter of the initial geometry is meshed. Quadratic cubic elements with 27 nodes for the kinematics and 4 nodes for the pressure are used (C27P4). The boundary condition and the load signal are given in figure 5 .

Figures 6(a), 6(b) and 6(c) show the global rate of convergence of the two proposed schemes: BETS and LBETS in comparison with a Runge-Kutta-Felhberg integrator (RKF). A relative numerical error is calculated from the global stress response at the end of the monotonic loading, using as a reference the numerical response obtained with a very small step size with each model $\left(\Delta t=10^{-4} s\right)$. The horizontal axis is the global size of the Newton increment used to solve the equilibrium equations. This global step-size does not always correspond exactly to the local integration stepsize, due to the sub-stepping. Two different tolerance values are used to control the sub-stepping strategy: $T O L=10^{-1}$ and $T O L=10^{-4}$. The following comments can be made about these curves:

- the RKF scheme has a global order of convergence of $O\left(\Delta t^{2}\right)$, except for the generalized Bingham model where the order of convergence is lower (near $O(\Delta t)$ ). This explicit scheme exhibits an irregular convergence with the Poynting Thomson model. The sub-stepping control tends to regularize the convergence, at least in the case of the Poynting Thomson model.

- the scalar Backward Euler scheme (BETS) has an order of convergence of $O(\Delta t)$, apart from the Poynting Thomson model, with which the convergence is lower. Due to is implicit nature, this scheme gives a more regular convergence than the RKF scheme. It seems to be less sensitive than the RKF scheme to the nonlinearity of the differential system. The sub-stepping control strategy has a direct impact on the global error, and it can be observed that the global relative error is influenced by the tolerance value.

- similar comments about the linearized scheme (LBETS) can be made: the order of convergence is approximately $O(\Delta t)$ and a regular convergence is observed. However, the global error is higher than with the other schemes. Apart from the Zener model, the linearization of the differential system near the trial elastic state appears to be a rather rough approximation. 


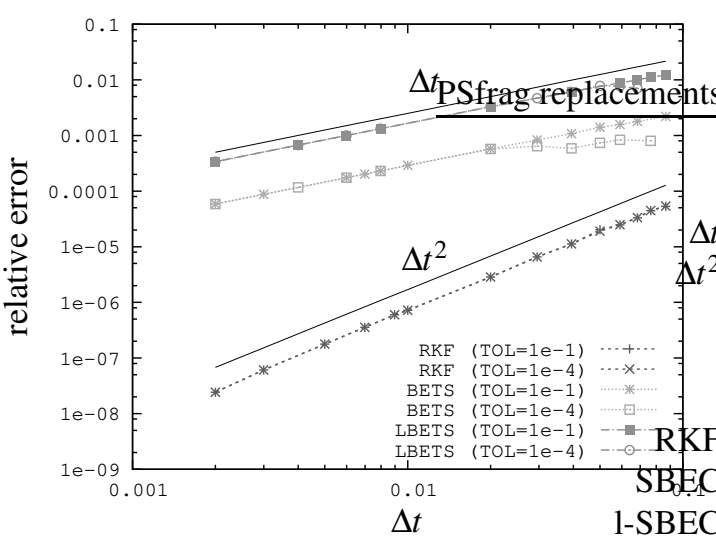

(a) Zener

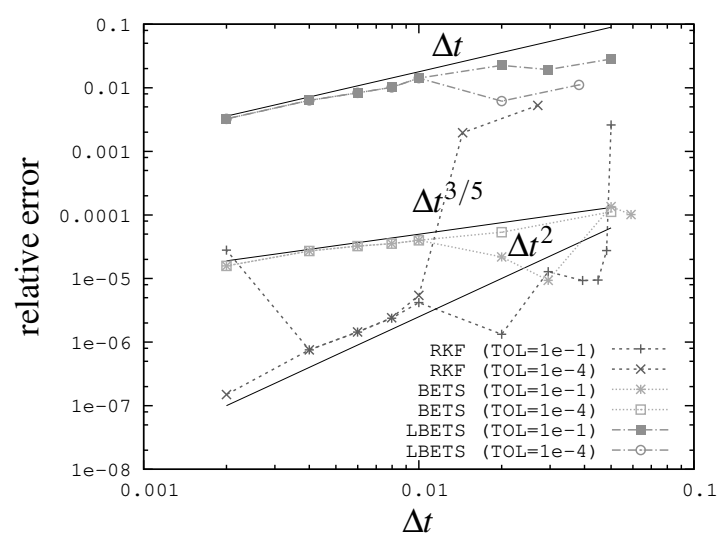

(b) Poynting-Thomson

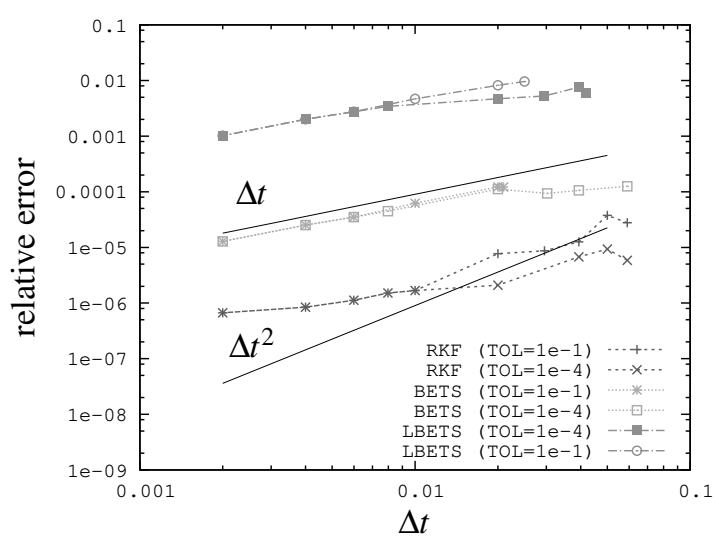

(c) Visco-Plastic

Figure 6: Order of convergence of each numerical integrator

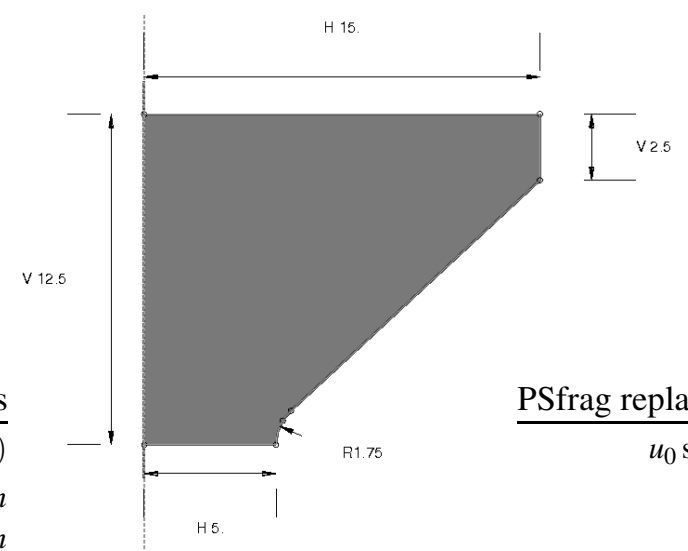

(a) geometry

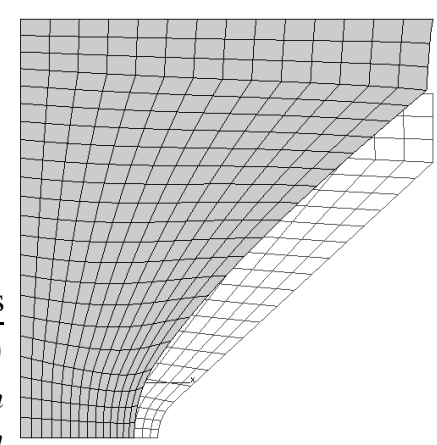

(b) deformed and undeformed mesh

Figure 7: Dumbbell specimen

\subsection{Cyclic traction on a dumbbell specimen}

A specimen consisting of one half of an axisymmetric dumbbell is (see figure 7) subjected to sinusoidal cyclic tensile loading with a tensile preload. The mesh comprises 308 quadrangle elements (Q9P3, quadratic displacement - 


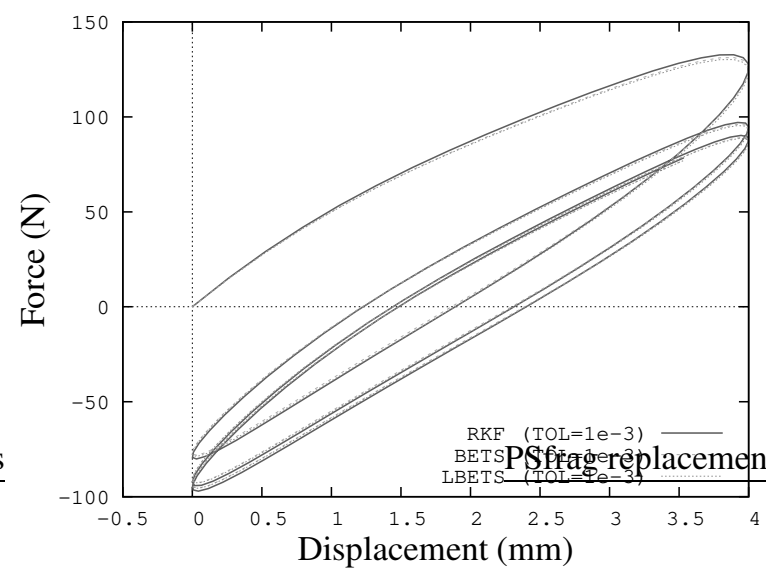

(a) Zener model

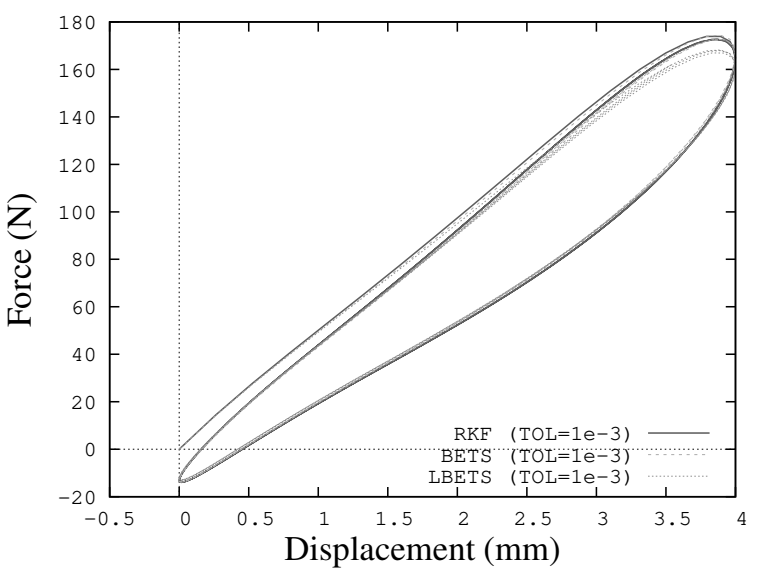

(b) Poynting-Thomson model

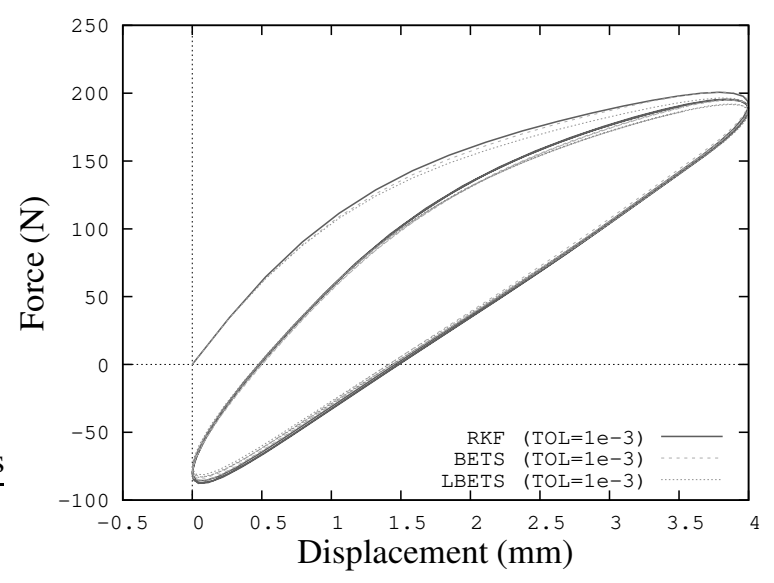

(c) Generelized-Bingham model

Figure 8: Cyclic traction test on a dumbbell specimen

linear pressure). The load signal applied is $u=u_{0}+u_{0} \sin (2 \pi f t)$, with $f=3 \mathrm{~Hz}, u_{0}=2.0 \mathrm{~mm}$.

Figure 8, gives the numerical results obtained with each scheme, with each rheological models. The initial global step-size was the same for each computation, and a value of $10^{-3}$ was used for the $T O L$ parameter. As in the previous case of homogeneous deformations, the following comments can be made:

- The results obtained with BETS and RKF schemes were very similar.

- The linearization of the elastic Cauchy-Green deformation near the trial state is an assumption which is too strong for complex non-linear flow rules. As shown is figures 8(b) and 8(c), the LBETS scheme did not fully reflect the non-linearity of the behavior.

Concerning the local results, figure 9 shows the Von-Mises stress obtained with the Poynting-Thomson model at two loading times. It can be seen from this figure that the RKF and BETS schemes gave very similar results on both loading and unloading stage. This was not the case of the linearized scheme, as can be seen by comparing figures 9(f) and $9(\mathrm{~d})$.

Table 2 sums up the numerical performance of each scheme by giving the total computing time, the total number of iterations and increments of Newton-Raphson required with each scheme. With complex non-linear flow rules (such as those involved in Poynting-Thomson and Generalized-Bingham models), BETS is a very interesting scheme 


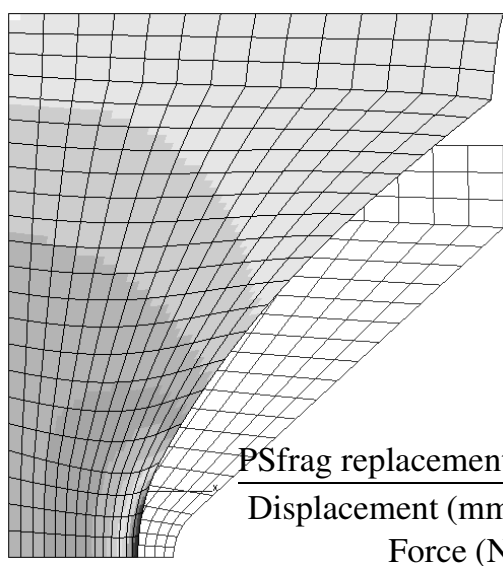

(a) RKF

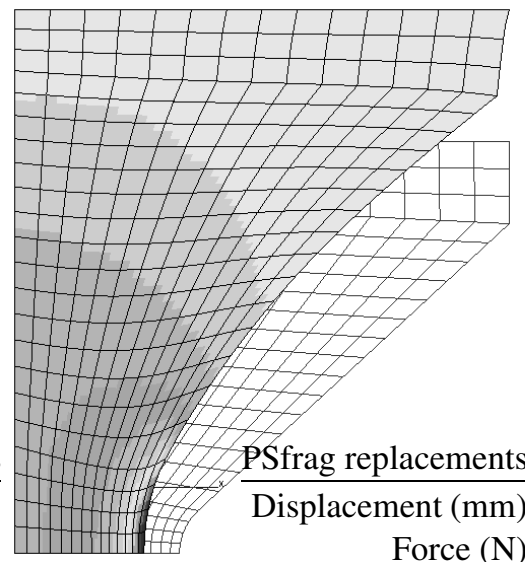

(b) BETS

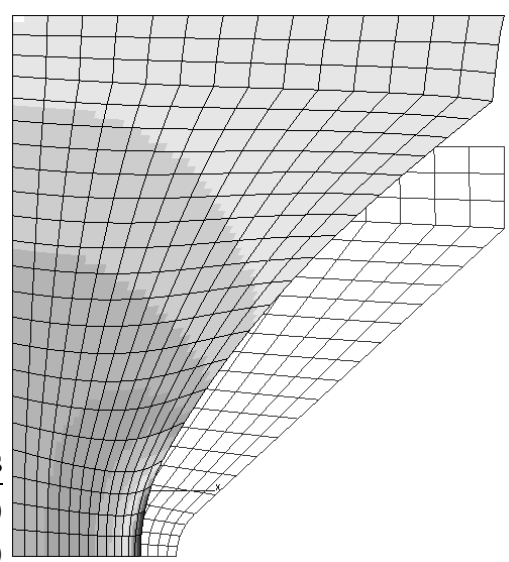

(c) LBETS

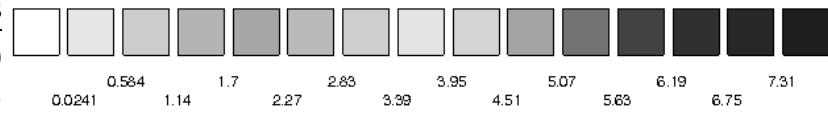

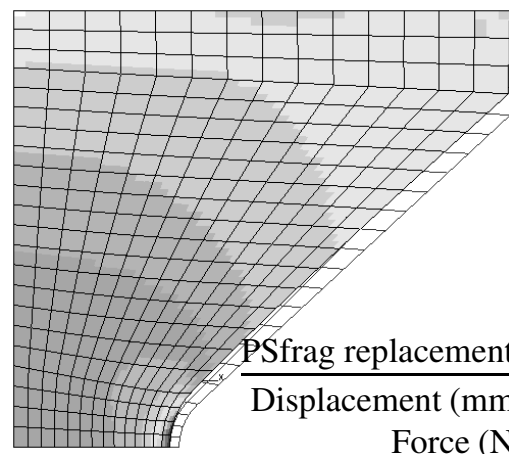

(d) RKF

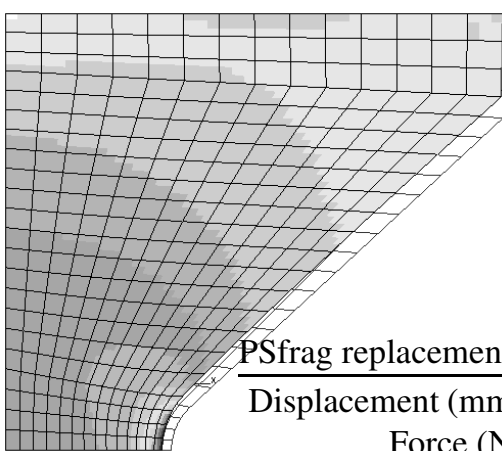

(e) BETS

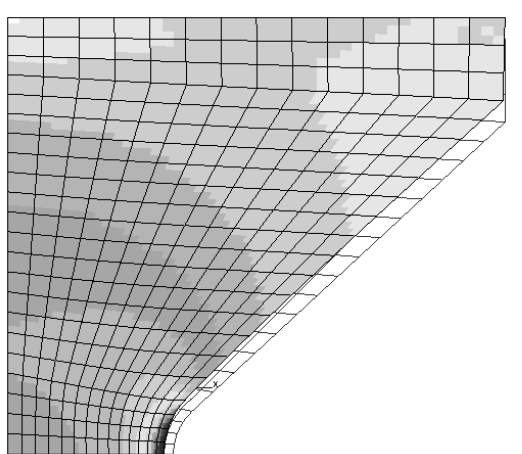

(f) LBETS

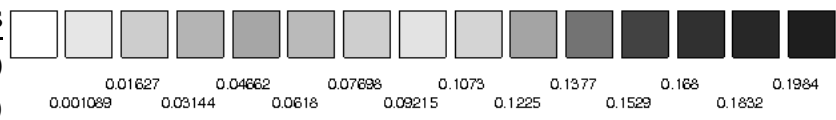

Figure 9: Von Mises stress for Poynting-Thomson model

as the total computing time is less or close than that required by a full tensorial scheme. In addition, the total number of iterations is lower than with the other schemes tested. This may be due to the tangent moduli, which is roughly approximated in the case of RKF. In the case of the Zener model, the RKF scheme was the most efficient as the total number of iterations was similar to that required with the BETS and the cost per iteration was lower with that of BETS. The linearization of BETS turned out to be less useful than expected. Although the resolution of a non linear system by a Newton scheme is avoided in the case of LBETS, computing an exponential matrix is time consuming (and this has to be done in both the flow rule computation and the tangent moduli calculation).

\section{Concluding comments}

Two integration schemes for finite viscoelasticity and finite viscoplasticity evolution laws are presented in this paper. As in the case of $\mathbf{J} 2$ finite elastoplasticity, the evolution equations are decomposed into flow direction and flow magnitude. The originality of the present approach is due to the fact that it focuses both on the choice of the flow direction, which depends directly on the flow rule and on the time stepping strategy, which is used to control 


\begin{tabular}{llcccc}
\hline Model & Integrator & TOL & Cpu time (s) & Nb Iter & Nb Incr \\
\hline Zener & RKF & $1 . \mathrm{e}-03$ & 86.5 & 478 & 158 \\
& BETS & $1 . \mathrm{e}-03$ & 130.4 & 476 & 158 \\
& LBETS & $1 . \mathrm{e}-03$ & 121.3 & 480 & 158 \\
\hline Poynting-Thomson & RKF & $1 . \mathrm{e}-03$ & 173.2 & 1088 & 158 \\
& BETS & $1 . \mathrm{e}-03$ & 166.2 & 929 & 203 \\
& LBETS & $1 . \mathrm{e}-03$ & 211.6 & 1402 & 179 \\
\hline Generalized-Bingham & RKF & $1 . \mathrm{e}-03$ & 102.3 & 657 & 158 \\
& BETS & $1 . \mathrm{e}-03$ & 118.0 & 491 & 158 \\
& LBETS & $1 . \mathrm{e}-03$ & 138.3 & 695 & 158 \\
\hline
\end{tabular}

Table 2: Numerical comparison of each integrator

the local deviation of the numerical solution. When applied to some simple examples this approach was found to be reliable in comparison with a classical Runge-Kutta scheme with three types of evolution laws. The BETS scheme was found to give the most satisfactory results, although it has the disadvantage of a weak rate of convergence, nearly 1 (which is also that of the exponential mapping scheme, see [13]). Its implicit aspect offers the advantage of a stable numerical behavior which was not the case of the explicit Runge-Kutta scheme used in this paper. In terms of its numerical performances, BETS is the most interesting scheme as it is not as sensitive to complex flow rules as the RKF scheme: the number of global Newton-Raphson iterations was smaller and therefore the computing time was shorter with BETS in the case of the Poynting-Thomson model.

Comparisons between the numerical results obtained with these schemes with three different rheological models showed that the Maxwell viscosity (at least with a quadratic pseudo-potential of dissipation) is not the most appropriate model for comparing numerical schemes. Although previous papers on this topic have focused on Maxwell rheological elements, this paper shows that Poynting-Thomson viscosity is a more interesting model to study the efficiency of a numerical integration scheme. It would therefore be a useful work comparing the use of exponential mapping method or DIRK method with this model.

To conclude, the numerical schemes presented in this paper are sufficiently generic to be implemented in standard (commercial) finite-element codes as they are defined at a local (Gauss points) level. On this basis, the numerical implementation of more complex rheological models can be easily achieved whenever the evolution equations can be written in the framework described in this paper.

\section{References}

[1] J. Simo, A framework for finite strain elastoplasticity based on maximum plastic dissipation and the multiplicative decomposition: Part ii. computational aspects, Computer Methods in Applied Mechanics and Enyineering 68 (1988) 1-31.

[2] J. Simo, T. Hughes, Computational Inelasticity, Springer-Verlag, 1998.

[3] S. Méo, A. Boukamel, . Débordes, Analysis of a thermoviscoelastic model in large strain, Computers \& Structures 80 (2002) $2085-2098$.

[4] L. Laiarinandrasana, R. Piques, A. Robisson, Visco-hyperelastic model with internal state variable coupled with discontinuous damage concept under total lagrangian formulation, International Journal of Plasticity 19 (2003) 977-1000.

[5] S. Hartmann, Computation in finite-strain viscoelasticity: finite elements based on the interpretation as differential-algebraic equations, Computer Methods in Applied Mechanics and Engineering 191 (2002) 1439-1470.

[6] G. Weber, L. Arnand, Finite deformation constitutive equations and a time integration procedure for isotropic hyperelastic-viscoplastic solids, Computer Methods in Applied Mechanics and Engineering 79 (1990) 173-202.

[7] S. Reese, S. Govindjee, A theory of finite viscoelasticity and numerical aspects, International Journal of Solid and Structures 35 (1998) 3455-3482.

[8] B. Nedjar, Frameworks for finite strain viscoelastic-plasticity based on multiplicative decompositions. part ii: Computational aspects, Computer Methods in Applied Mechanics and Engineering 191 (15-16) (2002) 1563-1593.

[9] E. Fancello, J. Vassoler, L. Stainier, A variational constitutive update algorithm for a set of isotropic hyperelastic-viscoplastic material models, Computer Methods in Applied Mechanics and Engineering 197 (2008) 4132-4148.

[10] P. Areias, K. Matouš, Finite element formulation for modeling nonlinear viscoelastic elastomers, Computer Methods in Applied Mechanics and Engineering 197 (2008) 4702-4717.

[11] H. Dal, M. Kaliske, Bergström-boyce model for nonlinear finite rubber viscoelasticity: theoretical aspects and algorithmic treatment for the fe method, Comput. Mech. 44 (2009) 809-823.

[12] F. Montans, K. Bathe, Computational issues in large strain elasto-plasticity: an algorithm for mixed hardening and plastic spin, International Journal for Numerical Methods in Engineering 63 (2005) 159-196. 
[13] A. Shutov, R. Kreiß ig, Finite strain viscoplasticity with nonlinear kinematic hardening: Phenomenological modeling and time integration, Computer Methods in Applied Mechanics and Engineering 197 (2008) 2015-2029.

[14] E. Fehlberg, Low-order classical runge-kutta formulas with step size control and their application to some heat transfer problems, Tech. rep., NASA TR R-315 (1969).

[15] M. K. Horn, Fourth and fifth-order, scaled runge-kutta algorithms for treating dense output, SIAM Journal on Numerical Analysis 20 (3) (1983) 558-568.

[16] F. Sidoroff, Un modéle viscoélastique non linéaire avec configuration intermédiaire, J. Méc. 13 (4) (1974) 679-713.

[17] F. Sidoroff, Variables internes en viscoélasticité, 2. milieux avec configuration intermédiaire, J. Méc. 14 (4) (1975) 571-595.

[18] R. Flory, Thermodynamic relations for highly elastic materials, Transactions of the Faraday Society 57 (1961) 829-838.

[19] M. Rüter, E. Stein, Analysis, finite element computation and error estimation in transversly isotropic nearly incompressible finite elasticity, Comput. Methods Appl. Mech. Engrg. 190 (2000) 519-541.

[20] J. Oden, N. Kikouchi, Finite element methods for constrained problems in elasticity, International Journal for Numerical Methods in Engineering 18 (1982) 701-725.

[21] T. Sussman, K. Bathe, A finite element formulation for nonlinear incompressible elastic and inelastic analysis, Computers \& Structures 26 (1987) 357-409.

[22] K. Bathe, Finite element procedures, Prentice Hall, 1996.

[23] M. Crisfield, Non-linear finite elements analysis of solids and structures, Wiley, 1996.

[24] S. Lejeunes, S. Méo, A. Boukamel, A direct numerical integration scheme for visco-hyperlastic models using radial return relaxation, European Journal of Computational Mechanics 19 (2010) 129-140.

[25] C. Moler, C. Van Loan, Ninteen dubious ways to compute the exponantial of a matrix, twenty-five years later, SIAM Review 45 (2003) 1-46.

[26] G. Holzapfel, Nonlinear Solid Mechanics, Wiley, 2004.

[27] S. Lejeunes, Modeling of laminated rubber-like/metal structures with a numerical reduction method, Ph.D. thesis, University of Aix-Marseille II (2006).

URL http://tel.archives-ouvertes.fr/tel-00090600/fr/

[28] R. Foerch, J. Besson, G. Cailletaud, P. Pivlin, Polymorphic constitutive equations in finite element codes, Comput. Methods Appl. Mech. Engrg. 141 (1996) 355-372.

[29] J. M. Martinez, Modelization and caracterization of hyper-visco-plastic behavior of a rubber-like material under multi-harmonic loadings and at various temperatures, Ph.D. thesis, University of Aix-Marseille II (2005).

URL http://tel.archives-ouvertes.fr/tel-00461986/fr/

\section{A. Scalar differential systems}

- Zener model:

Starting with:

$$
\boldsymbol{\sigma}_{n e q}=2 G \overline{\mathbf{B}}_{\mathbf{e}}^{\mathrm{D}}
$$

and introducing $\tau=4 G / J \eta$, the following system is obtained:

$$
\begin{cases}\frac{s-s^{\star}}{h} & =-\frac{\tau}{3} b^{2} \\ \frac{b-b^{\star}}{h} & =-\tau\left(\left(\mathbf{N}^{2}: \mathbf{N}\right) b^{2}+s b\right)\end{cases}
$$

- Poynting Thomson model:

The equilibrium stress is obtained by differentiating the free energy chosen:

$$
\boldsymbol{\sigma}_{e q}=2\left(C_{10}+3 C_{30}\left(I_{1}\left(\overline{\mathbf{B}}_{\mathbf{e}}\right)-3\right)^{2}\right) \overline{\mathbf{B}}_{\mathbf{e}}{ }^{D}-2 C_{01} \overline{\mathbf{B}}_{\mathbf{e}}^{-1^{\mathrm{D}}}
$$

where $\tau=4 G / J \eta, \tau_{1}=4 C_{10} / J \eta, \tau_{2}=4 C_{01} / J \eta, \tau_{3}=12 C_{30} / J \eta$ and the following quantities are introduced:

$$
\begin{aligned}
& \overline{\mathbf{B}}_{\mathbf{e}}{ }^{2}=b^{2} \mathbf{N}^{2}+s^{2} \mathbf{1}+2 s b \mathbf{N} \\
& I_{1}\left(\overline{\mathbf{B}}_{\mathbf{e}}\right)=\overline{\mathbf{B}}_{\mathbf{e}}: \mathbf{1}=3 s \\
& I_{2}\left(\overline{\mathbf{B}}_{\mathbf{e}}\right)=\frac{1}{2}\left(I_{1}^{2}\left(\overline{\mathbf{B}}_{\mathbf{e}}\right)-\left(\overline{\mathbf{B}}_{\mathbf{e}}{ }^{2}:(1)\right)\right)=3 s^{2}-\frac{b^{2}}{2}
\end{aligned}
$$

Using the Cailey-Hamilton theorem to evaluate $\overline{\mathbf{B}}_{\mathbf{e}}{ }^{-1}$, one has:

$$
\overline{\mathbf{B}}_{\mathbf{e}}{ }^{-1}=\overline{\mathbf{B}}_{\mathbf{e}}{ }^{2}-I_{1} \overline{\mathbf{B}}_{\mathbf{e}}+I_{2} \mathbf{1}=b^{2} \mathbf{N}^{2}-b s \mathbf{N}+\left(s^{2}-\frac{b^{2}}{2}\right) \mathbf{1}
$$


A more complex differential system that with the previous model is obtained

$$
\left\{\begin{aligned}
\frac{s-s^{\star}}{h} & =-\frac{\tau_{1}}{3} b^{2}+\left(\frac{\tau_{2} b^{2}}{9 s^{2}-\frac{3}{2} b^{2}}\right)\left(b\left(\mathbf{N}^{2}: \mathbf{N}\right)-s\right) \\
& +\frac{\tau}{3}(\overline{\mathbf{B}}: \mathbf{1})-\frac{\tau s}{3}\left(b^{2}\left(\overline{\mathbf{B}}: \mathbf{N}^{2}\right)\right. \\
& \left.-b s(\overline{\mathbf{B}}: \mathbf{N})+\left(s^{2}-\frac{b^{2}}{2}\right)(\overline{\mathbf{B}}: \mathbf{1})\right) \\
\frac{b-b^{\star}}{h} & =-\tau_{1}\left(\left(\mathbf{N}^{2}: \mathbf{N}\right) b^{2}+s b\right) \\
& +\left(\frac{\tau_{2} b}{3 s^{2}-\frac{1}{2} b^{2}}\right)\left(b^{2}\left(\mathbf{N}^{2}: \mathbf{N}^{2}\right)-s^{2}-\frac{b^{2}}{3}\right) \\
& +\tau(\overline{\mathbf{B}}: \mathbf{N})-\frac{\tau b}{3}\left(b^{2}\left(\overline{\mathbf{B}}: \mathbf{N}^{2}\right)\right. \\
& \left.-b s(\overline{\mathbf{B}}: \mathbf{N})+\left(s^{2}-\frac{b^{2}}{2}\right)(\overline{\mathbf{B}}: \mathbf{1})\right)
\end{aligned}\right.
$$

- Visco-Plastic model:

With this model, the solution of the evolution equations when $f\left(\boldsymbol{\sigma}_{n e q} \leq 0\right)$ is given exactly by the elastic trial state. When $f\left(\boldsymbol{\sigma}_{\text {neq }}>0\right)$, the system of equations is similar to that obtained with the Zener model:

$$
\left\{\begin{array}{l}
\frac{s-s^{\star}}{h}=-\frac{\tau}{3} b^{2}+\frac{2 \chi}{3 \eta} b \\
\frac{b-b^{\star}}{h}=-\tau\left(\left(\mathbf{N}^{2}: \mathbf{N}\right) b^{2}+s b\right)+\frac{2 \chi}{\eta}\left(\left(\mathbf{N}^{2}: \mathbf{N}\right) b+s\right)
\end{array}\right.
$$

\section{B. Tensorial operators}

The tensorial operators used in this paper are defined as follows:

\begin{tabular}{l|l}
$\cdot$ & the first order contracted tensorial product \\
\hline$:$ & the second order contracted tensorial product \\
\hline$\otimes$ & the classical tensorial product \\
\hline$\odot$ & $\begin{array}{l}\text { a second order tensorial operator } \\
\left([\mathbf{A} \ominus \mathbf{B}]_{i j k l}=A_{i l} B_{k j}\right)\end{array}$ \\
\hline$(2)$ & $\begin{array}{l}\text { a tensorial product between a fourth order tensor } \\
\text { and a second order tensor }\left([\mathbf{A}(2) \mathbf{B}]_{i j k l}=A_{i o k l} B_{o j}\right)\end{array}$ \\
\hline\|\| & $\begin{array}{l}\text { norm of a second order tensor } \\
\|\mathbf{A}\|=\sqrt{\mathbf{A}: \mathbf{A}}\end{array}$ \\
\hline$\bullet$ & $\begin{array}{l}\text { a tensorial product between a fourth order tensor } \\
\text { and a second order tensor }\left([\mathbf{A} \bullet \mathbf{B}]_{i j k l}=A_{i o k l} B_{o j}\right)\end{array}$
\end{tabular}

\section{An explicit integrator with step-size control: Runge-Kutta-Fehlberg}

Runge-Kutta schemes have been used by many authors because they are easy to implement and robust. Explicit versions (see [4]) and implicit ones (see [5]) have both been used. In this paper, we have adopted the explicit RungeKutta-Fehlberg method with which allow to control the step size in the numerical iterative scheme can be controlled via the error between a fourth and a fifth order Runge-Kutta scheme based on the same integration points ([14, 15] ). The main advantage of this scheme is that its numerical implementation is very simple.

The components of the tensor $\overline{\mathbf{B}}_{\mathbf{e}}$ are expressed in the vector form $\mathbf{Y}$ so as to be able to re-write the flow rule in eq. (16), (25) or (30) in the vector form:

$$
\dot{\mathbf{Y}}(t)=\mathcal{F}(\mathbf{Y}(t),\{\mathbf{L}(t)\},\{\mathbf{F}(t)\})
$$


Where the symbol $\{$.$\} denotes the vector form. The quantities depending on time, i.e. \mathbf{F}, J, \overline{\mathbf{B}}, \mathbf{L}$..., are evaluated at each integration point in each sub interval with respect to the hypothesis that $\mathbf{F}$ is linear with time. In the sub interval $\left[t_{k}, t_{k+1}\right], \mathbf{Y}_{k+1}$ is computed as follows:

$$
\left\{\begin{array}{l}
\mathbf{k}_{i}=\mathcal{F}\left(\mathbf{Y}_{k}+h \sum_{j=0}^{p} a_{i j} \mathbf{k}_{j},\left\{\mathbf{L}\left(t_{k}+c_{i} h\right)\right\},\left\{\mathbf{F}\left(t_{k}+c_{i} h\right)\right\}\right) \\
\mathbf{Y}_{k+1}=\mathbf{Y}_{k}+h \sum_{i=0}^{p} b_{i} \mathbf{k}_{i}
\end{array}\right.
$$

Where $h$ is the sub-interval size, $p$ is the order of the scheme, and $a_{i j}, b_{i}, c_{i}$ are given in the so-called Butcher-table (see table 3). The size of each sub-interval is adjusted so as to control the following local error:

$$
L E R=\left\|\mathbf{Y}_{k+1}^{[5]}-\mathbf{Y}_{k+1}^{[4]}\right\|
$$

Where $\mathbf{Y}_{k+1}^{[5]}$ is the solution vector of the 5th order scheme and $\mathbf{Y}_{k+1}^{[4]}$ is the solution vector of the 4th order one. Whenever this local error estimate satisfies $L E R<T O L$ the current step is accepted and the new step size is computed from

$$
h_{\text {new }}=0.9 \max \left(r_{\text {min }}, \min \left(r_{\text {max }},\left(\frac{T O L}{L E R}\right)^{1 / 3}\right)\right) h_{\text {old }}
$$
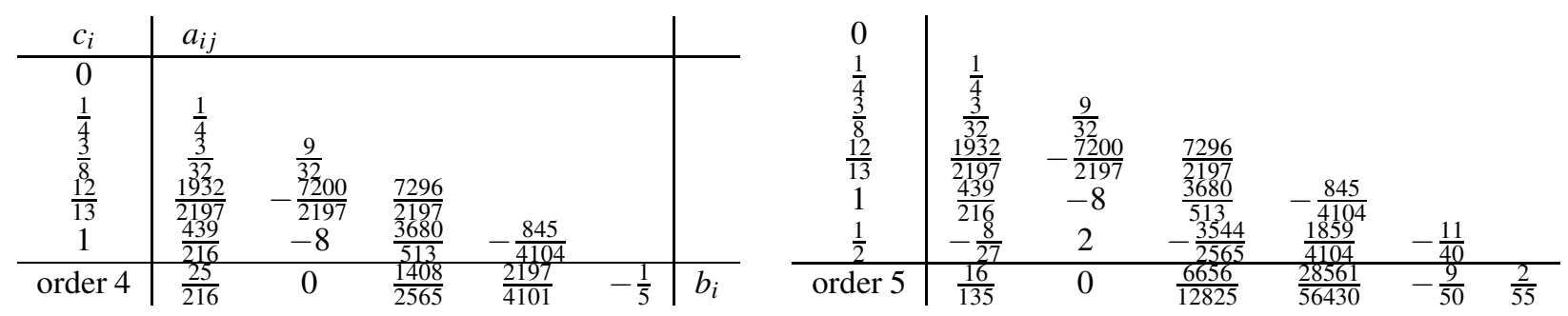

Table 3: Runge-Kutta-Fehlberg 4-5

As this integrator involves sub-discretisation, it is proposed to take only the elastic part of the tangent moduli by focusing on the trial configuration. For example, in the case of the Zener and Visco-plastic models ${ }^{6}$ where the isochoric part of the stress is composed of elastic and inelastic parts: $\tau_{i s o}=\tau_{e q}+\tau_{n e q}$, one has

$$
\frac{\partial \boldsymbol{\Pi}_{i s o}}{\partial \mathbf{F}}=\left(\tau_{e q}+\tau_{n e q}^{\star}\right) \bullet \frac{\partial \mathbf{F}^{-\mathrm{T}}}{\partial \mathbf{F}}+\left(\frac{\partial \tau_{e q}}{\partial \mathbf{F}}+\frac{\partial \tau_{n e q}^{\star}}{\partial \mathbf{F}}\right)\left(2 \mathbf{F}^{-\mathrm{T}}\right.
$$

Taking a general potential for the free energy depending on $I_{1}\left(\overline{\mathbf{B}}_{\mathbf{e}}\right)$ and $I_{2}\left(\overline{\mathbf{B}}_{\mathbf{e}}\right)$, the inelastic stress is given by

$$
\tau_{n e q}^{\star}=\frac{\partial \psi_{n e q}}{\partial I_{1}} \overline{\mathbf{B}}_{\mathbf{e}}^{\star^{\mathrm{D}}}+\frac{\partial \psi_{n e q}}{\partial I_{2}} \overline{\mathbf{B}}_{\mathbf{e}}^{\star^{-D}}
$$

and the computation of the differentiation of the stress with respect to $\mathbf{F}$ gives:

$$
\frac{\partial \tau_{n e q}^{\star}}{\partial \mathbf{F}}=\frac{\partial \tau_{n e q}^{\star}}{\partial \overline{\mathbf{B}}_{\mathbf{e}}^{\star}}: \frac{\partial \overline{\mathbf{B}}_{\mathbf{e}}^{\star}}{\partial \mathbf{F}}
$$

\footnotetext{
${ }^{6}$ The same applies to the Poynting Thomson model
} 
The first term in the latter expression follows from equation (80)

$$
\begin{aligned}
& \frac{\partial \tau_{n e q}^{\star}}{\partial \overline{\mathbf{B}}_{\mathbf{e}}{ }^{\star}}=\frac{\partial^{2} \psi_{n e q}}{\partial I_{1}^{2}} \overline{\mathbf{B}}_{\mathbf{e}}{ }^{{ }^{\mathrm{D}}} \otimes \mathbf{1}+\frac{\partial^{2} \psi_{n e q}}{\partial I_{2}^{2}} \overline{\mathbf{B}}_{\mathbf{e}}{ }^{\star^{-D}} \otimes\left(I_{1} \mathbf{1}-\overline{\mathbf{B}}_{\mathbf{e}}{ }^{\star}\right) \\
& +\frac{\partial^{2} \psi_{\text {neq }}}{\partial I_{1} I_{2}} \overline{\mathbf{B}}_{\mathbf{e}}{ }^{\mathrm{D}^{\mathrm{D}}} \otimes\left(I_{1} \mathbf{1}-\overline{\mathbf{B}}_{\mathbf{e}}{ }^{\star}\right)+\frac{\partial^{2} \psi_{\text {neq }}}{\partial I_{2} I_{1}} \overline{\mathbf{B}}_{\mathbf{e}}^{\star^{-D}} \otimes \mathbf{1} \\
& +\frac{\partial \psi_{\text {neq }}}{\partial I_{1}} \mathbb{P}-\frac{\partial \psi_{\text {neq }}}{\partial I_{2}}\left(\overline{\mathbf{B}}_{\mathbf{e}}^{\star^{-1}} \oplus \overline{\mathbf{B}}_{\mathbf{e}}^{\star^{-1}}-\frac{1}{3} \mathbf{1} \otimes \overline{\mathbf{B}}_{\mathbf{e}}^{\star^{-2}}\right)
\end{aligned}
$$

where $\mathbb{P}$ is the deviatoric operator: $\mathbb{P}=\mathbb{I}-(1 / 3) \mathbf{1} \otimes \mathbf{1}$. 